\title{
Rigidity of certain solvable actions on the sphere
}

\author{
MASAYUKi ASAOKA
}

\begin{abstract}
An analog of the Baumslag-Solitar group BS $(1, k)$ naturally acts on the sphere by conformal transformations. The action is not locally rigid in higher dimensions, but exhibits a weak form of local rigidity. More precisely, any perturbation preserves a smooth conformal structure.
\end{abstract}

$37 \mathrm{C} 85$

\section{Introduction}

Over the last two decades, it has been found that many smooth actions of discrete groups exhibit local rigidity. Most known examples are classified into two classes:

(1) Anosov or partially hyperbolic $\mathbb{Z}^{n}$-actions and homogeneous actions of cocompact lattices related to Anosov or partially hyperbolic $\mathbb{R}^{n}$-actions with $n \geq 2$ (see Damjanović and Katok [4], Katok and Lewis [12], Katok and Spatzier [13] and Niţică and Török [16]).

(2) Isometric or quasiaffine actions of lattices or groups with Property (T) (see Benveniste [1], Fisher and Margulis [7; 8] and Zimmer [19]).

See Fisher's survey [6] for more related results.

One of the exceptions is an action of the Baumslag-Solitar group $\mathrm{BS}(1, k)$ on the circle. For $k \geq 2$, the Baumslag-Solitar group $\mathrm{BS}(1, k)$ is a finitely presented solvable group defined by $\mathrm{BS}(1, k)=\left\langle a, b \mid a b a^{-1}=b^{k}\right\rangle$. It is isomorphic to a group generated by two affine transformations of the real line: $f(x)=k x$ and $g(x)=x+c$ with $c \neq 0$. The natural extensions of $f$ and $g$ to $S^{1}=\mathbb{R} \cup\{\infty\}$ define a real analytic action $\rho_{c}$ of $\mathrm{BS}(1, k)$ on $S^{1}$. Note $\rho_{c}$ is conjugate to $\rho_{1}$ by a diffeomorphism $h(x)=c^{-1} x$.

Theorem 1.1 (Burslem and Wilkinson [2]) Any real analytic action of BS( $1, k)$ on the circle is locally rigid. In particular, the action $\rho_{c}$ is locally rigid. 
In the same paper, Burslem and Wilkinson also gave a smooth classification of $C^{r}$ actions of BS $(1, k)$ on $S^{1}$ by using Navas' complete topological classification of $C^{2}$ solvable actions on one-dimensional manifolds [15]. Guelman and Liousse [10] extended the classification by Burslem and Wilkinson to $C^{1}$ actions by using Cantwell and Conlon's work [3] on $C^{1}$ actions of $\mathrm{BS}(1, k)$ on the circle or a closed interval and Rivas' work [17] on $C^{0}$ action of $\mathrm{BS}(1, k)$ on the real line.

Recently, some people have studied actions of Baumslag-Solitar like groups on higher dimensional manifolds. McCarthy [14] proved the rigidity of trivial actions of a large class of abelian-by-cyclic groups on an arbitrary dimensional closed manifold. Guelman and Liousse [9] studied actions of $\mathrm{BS}(1, k)$ on surfaces and gave a $C^{\infty}$ faithful action on the 2-torus which is not locally rigid even in a topological sense.

In this paper, we study a natural higher dimensional analog of the standard $\mathrm{BS}(1, k)-$ action $\rho_{c}$. For $n \geq 1$ and $k \geq 2$, we define a finitely generated solvable group $\Gamma_{k, n}$ by

$$
\left.\Gamma_{n, k}=\left\langle a, b_{1}, \ldots, b_{n}\right| a b_{i} a^{-1}=b_{i}^{k}, b_{i} b_{j}=b_{j} b_{i} \text { for any } i, j=1, \ldots, n\right\rangle .
$$

The group $\Gamma_{n, k}$ admits a natural action on the $n$-dimensional sphere $S^{n}$. We identify $S^{n}$ with $\mathbb{R}^{n} \cup\{\infty\}$ by the stereographic projection. For any basis $B=\left(v_{1}, \ldots, v_{n}\right)$ of $\mathbb{R}^{n}$, define a $\Gamma_{n, k}$-action $\rho_{B}$ on $S^{n}$ by

- $\rho_{B}^{a}(x)=k x$ and $\rho_{B}^{b_{i}}(x)=x+v_{i}$ for $x \in \mathbb{R}^{n}=S^{n} \backslash\{\infty\}$,

- $\rho_{B}^{a}(\infty)=\rho_{B}^{b_{i}}(\infty)=\infty$.

The action $\rho_{B}$ preserves the standard conformal structure on $S^{n}$ and we call it the standard action associated to $B$. For $n=1$ and $v_{1}=c \neq 0$, the group $\Gamma_{1, k}$ is the Baumslag-Solitar group $\mathrm{BS}(1, k)$ and the action $\rho_{B}$ is the standard action $\rho_{c}$. Therefore, $\rho_{B}$ is locally rigid by Theorem 1.1 if $n=1$. On the other hand, $\rho_{B}$ is not locally rigid for any basis $B$ if $n \geq 2$ (see Proposition 3.1). Hence, a direct analog of Theorem 1.1 does not hold.

The aim of this paper is to show that the action $\rho_{B}$ exhibits a weak form of local rigidity for $n \geq 2$.

To state the main theorem, we recall basic concepts on rigidity of group actions. Let $\Gamma$ be a discrete group and $G$ a topological group. $\operatorname{By} \operatorname{Hom}(\Gamma, G)$, we denote the set of homomorphisms from $\Gamma$ to $G$. For $\rho \in \operatorname{Hom}(\Gamma, G)$ and $\gamma \in \Gamma$, we put $\rho^{\gamma}=\rho(\gamma)$. The set $\operatorname{Hom}(\Gamma, G)$ is naturally identified with a subset of the power set $G^{\Gamma}$. The product topology on $G^{\Gamma}$ induces a topology on $\operatorname{Hom}(\Gamma, G)$. When $G$ is Hausdorff, a sequence $\left(\rho_{m}\right)_{m \geq 1}$ in $\operatorname{Hom}(\Gamma, G)$ converges to $\rho$ if and only if $\rho_{m}^{\gamma}$ converges to $\rho^{\gamma}$ for any $\gamma \in \Gamma$. 
Let $M$ be a smooth closed manifold. Below, all smooth maps and diffeomorphisms are of class $C^{\infty}$. By $\operatorname{Diff}(M)$, we denote the group of diffeomorphisms of $M$. It naturally becomes a topological group by the $C^{\infty}$-topology. For a discrete group $\Gamma$, a smooth left $\Gamma$-action on $M$ is just a homomorphism from $\Gamma$ to $\operatorname{Diff}(M)$. Hence, $\operatorname{Hom}(\Gamma, \operatorname{Diff}(M))$ is identified with the space of (smooth left) $\Gamma$-actions on $M$. We say that two actions $\rho_{1} \in \operatorname{Hom}\left(\Gamma, \operatorname{Diff}\left(M_{1}\right)\right)$ and $\rho_{2} \in \operatorname{Hom}\left(\Gamma, \operatorname{Diff}\left(M_{2}\right)\right)$ are smoothly conjugate if there exists a diffeomorphism $h: M_{1} \rightarrow M_{2}$ such that $\rho_{2}^{\gamma} \circ h=h \circ \rho_{1}^{\gamma}$ for any $\gamma \in \Gamma$. We also say that an action $\rho_{0} \in \operatorname{Hom}(\Gamma, \operatorname{Diff}(M))$ is locally rigid if there exists a neighborhood $\mathcal{U}$ of $\rho_{0}$ in $\operatorname{Hom}(\Gamma, \operatorname{Diff}(M))$ such that any action $\rho$ in $\mathcal{U}$ is smoothly conjugate to $\rho_{0}$.

Now, we are ready to state the main theorem of this paper.

Main Theorem Suppose $n, k \geq 2$. Let $\rho_{B}$ be the standard $\Gamma_{n, k}$-action on $S^{n}$ associated to a basis $B$ of $\mathbb{R}^{n}$. Then, there exists a neighborhood $\mathcal{U} \subset \operatorname{Hom}\left(\Gamma_{n, k}, \operatorname{Diff}\left(S^{n}\right)\right)$ of $\rho_{B}$ such that any $\rho \in \mathcal{U}$ is smoothly conjugate to $\rho_{B^{\prime}}$ for some basis $B^{\prime}=B^{\prime}(\rho)$ of $\mathbb{R}^{n}$. In particular, any action in $\mathcal{U}$ preserves a $C^{\infty}$ conformal structure of $S^{n}$.

The proof is divided into three steps: First, we show a local version of the main theorem, ie, rigidity of $\rho_{B}$ as a local action at $\infty$. This is the main step of the proof. Second, we prove that any perturbation of $\rho_{B}$ admits a global fixed point near $\infty$. Finally, we extend the local conjugacy obtained in the first step to a global one.

The strategy for the first step is close to Burslem and Wilkinson's one in [2]. However, there is an essential difference from their case; the action $\rho_{B}$ admits nontrivial deformation. The difficulty is that there seems no direct way to find a basis $B^{\prime}=B^{\prime}(\rho)$ such that $\rho$ is conjugate to $\rho_{B^{\prime}}$ for a given perturbation $\rho$ of $\rho_{B}$. To overcome it, we follow Weil's idea in [18], where he controlled deformation of lattices of Lie groups by the first cohomology of a deformation complex. Note that Benveniste [1] and Fisher [5] proved local rigidity of isometric actions by applying Weil's idea to $\operatorname{Hom}(\Gamma, \operatorname{Diff}(M))$. In their cases, the deformation complex is infinite dimensional, and hence, they needed Hamilton's Implicit Function Theorem for tame maps between Fréchet spaces. In our case, we reduce the deformation complex to a finite dimensional one and Weil's Implicit Function Theorem is sufficient.

In [2], Burslem and Wilkinson gave another proof of the first step above for BS( $1, k)-$ actions on $S^{1}$. They showed the existence of an invariant projective structure on a neighborhood of the global fixed point by using the Schwarzian derivative. The author does not know whether there is an analogous proof for higher dimensional case. Finding it seems an interesting problem. 
Acknowledgements The author would like to thank an anonymous referee for valuable comments. The author is partially supported by JSPS Grant-in-Aid for Young Scientists (A).

\section{Proof of Main Theorem}

\subsection{Local version of the main theorem}

Let $M_{n}(\mathbb{R})$ be the set of real square matrices of size $n$ and $\mathrm{GL}_{n}(\mathbb{R})$ be the group of invertible matrices in $M_{n}(\mathbb{R})$. We identify each element of $M_{n}(\mathbb{R})$ with an $n$-tuple of column vectors in $\mathbb{R}^{n}$. Under this identification, $\mathrm{GL}_{n}(\mathbb{R})$ is the set of bases of $\mathbb{R}^{n}$. By $\|\cdot\|$, we denote the Euclidean norm of $\mathbb{R}^{n}$. Let $\mathcal{S}^{r}\left(\mathbb{R}^{n}\right)$ be the set of symmetric $r$-multilinear maps from $\left(\mathbb{R}^{n}\right)^{r}$ to $\mathbb{R}^{n}$. We define a norm $\|\cdot\|^{(r)}$ on $\mathcal{S}^{r}\left(\mathbb{R}^{n}\right)$ by

$$
\|F\|^{(r)}=\sup \left\{\left\|F\left(\xi_{1}, \ldots, \xi_{r}\right)\right\| \mid \xi_{1}, \ldots, \xi_{r} \in \mathbb{R}^{n},\left\|\xi_{i}\right\| \leq 1 \text { for any } i\right\} .
$$

Note $\left\|F\left(\xi_{1}, \ldots, \xi_{r}\right)\right\| \leq\|F\| \cdot\left\|\xi_{1}\right\| \cdots\left\|\xi_{r}\right\|$ for any $\xi_{1}, \ldots, \xi_{r} \in \mathbb{R}^{n}$ and $\|A\|^{(1)}$ is the operator norm of $A \in M_{n}(\mathbb{R})=\mathcal{S}^{1}\left(\mathbb{R}^{n}\right)$.

Let $\mathcal{D}\left(\mathbb{R}^{n}, 0\right)$ be the group of germs of local diffeomorphisms of $\mathbb{R}^{n}$ at the origin. For $F \in \mathcal{D}\left(\mathbb{R}^{n}, 0\right)$, we denote the $r$-th derivative of $F$ at the origin by $D_{0}^{(r)} F$. It is an element of $\mathcal{S}^{r}\left(\mathbb{R}^{n}\right)$. For $r \geq 2$, define the $C_{\text {loc }}^{r}$-topology on $\mathcal{D}\left(\mathbb{R}^{n}, 0\right)$ by a pseudodistance $d_{C_{\mathrm{loc}}^{r}}(F, G)=\sum_{i=1}^{r}\left\|D_{0}^{(i)} F-D_{0}^{(i)} G\right\|^{(i)}$. Notice that $d_{C_{\mathrm{loc}}^{r}}$ is not a distance, and hence, the $C_{\text {loc }}^{r}$-topology is not Hausdorff.

For a discrete group $\Gamma$, the $C_{\text {loc }}^{r}$-topology on $\operatorname{Hom}\left(\Gamma, \mathcal{D}\left(\mathbb{R}^{n}, 0\right)\right)$ is naturally introduced as before. We say two local actions $P_{1}, P_{2} \in \operatorname{Hom}\left(\Gamma, \mathcal{D}\left(\mathbb{R}^{n}, 0\right)\right)$ are smoothly conjugate if there exists $H \in \mathcal{D}\left(\mathbb{R}^{n}, 0\right)$ such that $P_{2}^{\gamma} \circ H=H \circ P_{1}^{\gamma}$ for any $\gamma \in \Gamma$.

Let $\bar{\phi}$ be a diffeomorphism from $S^{n} \backslash\{0\}$ to $\mathbb{R}^{n}$ given by

$$
\bar{\phi}(x)=\frac{1}{\|x\|^{2}} \cdot x .
$$

For $B \in M_{n}(\mathbb{R})$, we define a local action $P_{B} \in \operatorname{Hom}\left(\Gamma_{n, k}, \mathcal{D}\left(\mathbb{R}^{n}, 0\right)\right)$ by

$$
P_{B}^{\gamma}=\bar{\phi} \circ \rho_{B}^{\gamma} \circ \bar{\phi}^{-1} \text {. }
$$

In this subsection, we prove the following local version of the main theorem.

Theorem 2.1 For $B \in \mathrm{GL}_{n}(\mathbb{R})$, there exists a $C_{\text {loc }}^{2}$-neighborhood $\mathcal{U}$ of $P_{B}$ such that any local action $P \in \mathcal{U}$ is smoothly conjugate to $P_{B^{\prime}}$ for some $B^{\prime}=B^{\prime}(P) \in \mathrm{GL}_{n}(\mathbb{R})$. 
The proof is divided into several steps. First, we show the stability of the linear part of $P^{b_{i}}$. Let $\bar{F}$ be the element of $\mathcal{D}\left(\mathbb{R}^{n}, 0\right)$ given by

$$
\bar{F}(x)=k^{-1} x .
$$

Notice that $P_{B}^{a}=\bar{F}$ and $D_{0}^{(1)} P_{B}^{b_{i}}=I$ for any $B \in M_{n}(\mathbb{R})$ and $i=1, \ldots, n$.

Lemma 2.2 Let $m$ be a positive integer and $P_{*}$ a local action in $\operatorname{Hom}\left(\Gamma_{n, k}, \mathcal{D}\left(\mathbb{R}^{m}, 0\right)\right)$. Suppose that $D_{0}^{(1)} P_{*}^{a}=k^{-1} I$ and $D_{0}^{(1)} P_{*}^{b_{i}}=I$ for any $i=1, \ldots, n$. Then, there exists a $C_{0}^{1}$-neighborhood $\mathcal{U}$ of $P_{*}$ in $\operatorname{Hom}\left(\Gamma_{n, k}, \mathcal{D}\left(\mathbb{R}^{m}, 0\right)\right)$ such that $D_{0}^{(1)} P^{b_{i}}=I$ for any $P \in \mathcal{U}$ and $i=1, \ldots, n$.

Proof Put $c_{k j}=k ! /(j !(k-j) !)$. There exists $\delta>0$ such that

$$
\delta \cdot\left(k+k^{2}+\sum_{j=2}^{k} c_{k j} \delta^{j-2}(1+k \delta)\right) \leq \frac{1}{2} .
$$

Take a $C_{\mathrm{loc}}^{1}$-neighborhood $\mathcal{U}$ of $P_{*}$ such that $\left\|D_{0}^{(1)} P^{\gamma}-D_{0}^{(1)} P_{*}^{\gamma}\right\|<\delta$ for any $P \in \mathcal{U}$ and $\gamma=a, b_{1}, \ldots, b_{m}$. Fix $P \in \mathcal{U}$ and $i=1, \ldots, n$. Let $A=D_{0}^{(1)} P^{a}-D_{0}^{(1)} P_{*}^{a}$ and let $B=D_{0}^{(1)} P^{b_{i}}-D_{0}^{(1)} P_{*}^{b_{i}}$. We need to show that $B=0$. By the equalities $D_{0}^{(1)} P_{*}^{a}=$ $k^{-1} I, D_{0}^{(1)} P_{*}^{b_{i}}=I$ and $P^{a} \circ P^{b_{i}}=P^{b_{i}^{k}} \circ P^{a}$, we have $\left(k^{-1} I+A\right)(I+B)=$ $(I+B)^{k}\left(k^{-1} I+A\right)$. Hence,

$$
\begin{aligned}
(k-1)\|B\|^{(1)} & =\left\|k A B-k^{2} B A-\sum_{j=2}^{k} c_{k j} B^{j}(I+k A)\right\|^{(1)} \\
& \left.\leq\left(k \delta+k^{2} \delta+\sum_{j=2}^{k} c_{k j} \delta^{j-1}(1+k \delta)\right)\right) \cdot\|B\|^{(1)} \\
& \leq \frac{1}{2} \cdot\|B\|^{(1)} .
\end{aligned}
$$

Since $k \geq 2$, we obtain that $B=0$.

Second, we show the stability of the linear part of $P^{a}$. Let $\langle\cdot, \cdot\rangle$ be the Euclidean inner product of $\mathbb{R}^{n}$. For $v \in \mathbb{R}^{n}$, we define $Q_{v} \in \mathcal{S}^{2}\left(\mathbb{R}^{n}\right)$ by

$$
Q_{v}(\xi, \eta)=\langle\xi, \eta\rangle \cdot v-\langle\xi, v\rangle \cdot \eta-\langle\eta, v\rangle \cdot \xi
$$

By a direct calculation, we can check that

$$
D_{0}^{(2)} P_{B}^{b_{i}}=2 Q_{v_{i}}
$$

for any $B=\left(v_{1}, \ldots, v_{n}\right) \in M_{n}(\mathbb{R})$ and $i=1, \ldots, n$. 
Lemma 2.3 For any given $B \in \mathrm{GL}_{n}(\mathbb{R})$, there exists a $C_{0}^{2}$-neighborhood $\mathcal{U}$ of $P_{B}$ in $\operatorname{Hom}\left(\Gamma_{n, k}, \mathcal{D}\left(\mathbb{R}^{n}, 0\right)\right)$ such that $D_{0}^{(1)} P^{a}=k^{-1} I$ for any $P \in \mathcal{U}$.

Proof For any $F, G \in \mathcal{D}\left(\mathbb{R}^{n}, 0\right)$ with $D_{0}^{(1)} G=I$, it is easy to see that

$$
\begin{aligned}
D_{0}^{(2)}(F \circ G) & =D_{0}^{(2)} F+D_{0}^{(1)} F \circ D_{0}^{(2)} G, \\
D_{0}^{(2)}\left(G^{k} \circ F\right) & =D_{0}^{(2)} F+k \cdot D_{0}^{(2)} G \circ\left(D_{0}^{(1)} F, D_{0}^{(1)} F\right) .
\end{aligned}
$$

Put $B=\left(v_{1}, \ldots, v_{n}\right)$. Since $B=\left(v_{1}, \ldots, v_{n}\right)$ is a basis of $\mathbb{R}^{n}$, there is a constant $\epsilon>0$ such that $\max _{i=1, \ldots, n}\left\{\left\|A^{\prime} v_{i}\right\|\right\} \geq \epsilon\left\|A^{\prime}\right\|^{(1)}$ for any $A^{\prime} \in M_{n}(\mathbb{R})$. By Lemma 2.2, there exists a $C_{\text {loc }}^{1}$-neighborhood $\mathcal{U}_{1}$ of $P_{B}$ such that $D_{0}^{(1)} P^{b_{i}}=I$ for any $P \in \mathcal{U}_{1}$. Let $\mathcal{U}$ be a $C_{\text {loc }}^{2}$-open neighborhood of $P_{B}$ consisting of $P \in \mathcal{U}_{1}$ such that

$$
\max _{i=1, \ldots, n}\left\{3\left\|D_{0}^{(2)} P^{b_{i}}-2 Q_{v_{i}}\right\|^{(2)}+\left\|k \cdot D_{0}^{(1)} P^{a}-I\right\| \cdot\left\|D_{0}^{(2)} P^{b_{i}}\right\|^{(2)}\right\}<\epsilon .
$$

Fix $P \in \mathcal{U}_{1}$ and put

$$
\begin{aligned}
A & =k \cdot D_{0}^{(1)} P^{a}-I, \\
B_{i} & =D_{0}^{(2)} P^{b_{i}}-D_{0}^{(2)} P_{B}^{b_{i}}=D_{0}^{(2)} P^{b_{i}}-2 Q_{v_{i}}, \\
C_{i} & =A \circ Q_{v_{i}}-2 Q_{v_{i}} \circ(A, I) .
\end{aligned}
$$

We will show that $A=0$. Since $P^{a} \circ P^{b_{i}}=P^{b_{i}^{k}} \circ P^{a}$, we have

$$
k^{-1}(I+A) \circ\left(2 Q_{v_{i}}+B_{i}\right)=k \cdot\left(2 Q_{v_{i}}+B_{i}\right) \circ\left(k^{-1}(I+A), k^{-1}(I+A)\right) .
$$

It implies that

$$
\begin{aligned}
2\left\|C_{i}\right\|^{(2)} & =\left\|A \circ B_{i}-2 B_{i} \circ(A, I)-\left(2 Q_{v_{i}}+B_{i}\right) \circ(A, A)\right\|^{(2)} \\
& \leq\|A\|^{(1)} \cdot\left(3\left\|B_{i}\right\|^{(2)}+\|A\|^{(1)} \cdot\left\|D_{0}^{(2)} P^{b_{i}}\right\|^{(2)}\right) \\
& \leq \epsilon\|A\|^{(1)}
\end{aligned}
$$

for any $i=1, \ldots, n$. The definition of $Q_{v}$ also implies $C_{i}\left(v_{i}, v_{i}\right)=\left\|v_{i}\right\|^{2} \cdot A v_{i}$, and hence, $\left\|C_{i}\right\|^{(2)} \geq\left\|A v_{i}\right\|$. Therefore, we obtain

$$
2 \epsilon\|A\|^{(1)} \leq 2 \max _{i=1, \ldots, n}\left\|A v_{i}\right\| \leq \epsilon\|A\|^{(1)} .
$$

It implies that $A=0$, and hence, $D_{0}^{(1)} P^{a}=k^{-1} \cdot I$.

Let $\mathcal{M}_{1}{ }^{\prime}$ be the subset of $\operatorname{Hom}\left(\Gamma_{n, k}, \mathcal{D}\left(\mathbb{R}^{n}, 0\right)\right)$ consisting of local actions $P$ such that $P^{a}=\bar{F}$ and $D_{0}^{(1)} P^{b_{i}}=I$ for any $i=1, \ldots, n$. Notice that $P_{B}$ is an element of $\mathcal{M}_{1}^{\prime}$ for any $B \in \mathrm{GL}_{n}(\mathbb{R})$. 
Proposition 2.4 Let $B$ be an element of $\mathrm{GL}_{n}(\mathbb{R})$. For any $C_{\mathrm{loc}}^{2}-$ neighborhood $\mathcal{U}_{0}$ of $P_{B}$ in $\operatorname{Hom}\left(\Gamma_{n, k}, \mathcal{D}\left(\mathbb{R}^{n}, 0\right)\right)$, there exists another $C_{\text {loc }}^{2}-$ neighborhood $\mathcal{U}$ of $P_{B}$ such that any $P \in \mathcal{U}$ is smoothly conjugate to a local action in $\mathcal{U}_{0} \cap \mathcal{M}_{1}{ }^{\prime}$.

Proof By Lemmas 2.2 and 2.3, there exists a $C_{\text {loc }}^{2}$-neighborhood $\mathcal{U}_{1}$ of $P_{B}$ in $\operatorname{Hom}\left(\Gamma_{n, k}, \mathcal{D}\left(\mathbb{R}^{n}, 0\right)\right)$ such that $D_{0}^{(1)} P^{a}=k^{-1} \cdot I$ and $D_{0}^{(1)} P^{b_{i}}=I$ for any $P \in \mathcal{U}_{1}$ and $i=1, \ldots, n$. Fix $P \in \mathcal{U}_{1}$. It is known that if a local diffeomorphism $F \in \mathcal{D}\left(\mathbb{R}^{n}, 0\right)$ satisfies $D_{0}^{(1)} F=\alpha I$ for some $0<\alpha<1$, then it is smoothly linearizable (see [11, Theorem 6.6.6]). Hence, there exists $H \in \mathcal{D}\left(\mathbb{R}^{n}, 0\right)$ such that $D_{0}^{(1)} H=I$ and $\bar{F}=H \circ P^{a} \circ H^{-1}$. We define a local action $P^{H} \in \operatorname{Hom}\left(\Gamma_{n, k}, \mathcal{D}\left(\mathbb{R}^{n}, 0\right)\right)$ by $\left(P^{H}\right)^{\gamma}=H \circ P^{\gamma} \circ H^{-1}$. Since $D_{0}^{(1)}\left(P^{H}\right)^{b_{i}}=D_{0}^{(1)} P^{b_{i}}=I$, the local action $P^{H}$ is contained in $\mathcal{M}_{1}{ }^{\prime}$. From the equation $D_{0}^{(2)}(H \circ \bar{F})=D_{0}^{(2)}\left(P^{a} \circ H\right)$, we obtain $(k-1) D_{0}^{(2)} H=k^{2} D_{0}^{(2)} P^{a}$. Hence, there exists a small $C_{\text {loc }}^{2}-$ neighborhood $\mathcal{U} \subset \mathcal{U}_{1}$ of $P_{B}$ such that $P^{H} \in \mathcal{U}_{0}$ for any $P \in \mathcal{U}$.

Following Weil's idea, we reduce Theorem 2.1 to exactness of a linear complex. Put

$$
\begin{aligned}
& \mathcal{M}_{0}=\operatorname{GL}_{n}(\mathbb{R}) \times \operatorname{GL}_{n}(\mathbb{R}), \\
& \mathcal{M}_{1}=\left\{\left(G_{i}\right)_{1 \leq i \leq n} \in \mathcal{D}\left(\mathbb{R}^{n}, 0\right)^{n} \mid D_{0}^{(1)} G_{i}=I, \bar{F} \circ G_{i}=G_{i}^{k} \circ \bar{F} \text { for any } i\right\}, \\
& \mathcal{M}_{2}=\left\{\left(C_{i j}\right)_{1 \leq i<j \leq n} \mid C_{i j} \in \mathcal{S}^{3}\left(\mathbb{R}^{n}\right)\right\}=\left(\mathcal{S}^{3}\left(\mathbb{R}^{n}\right)\right)^{n(n-1) / 2} .
\end{aligned}
$$

Define maps $\Phi: \mathcal{M}_{0} \rightarrow \mathcal{M}_{1}$ and $\Psi: \mathcal{M}_{1} \rightarrow \mathcal{M}_{2}$ by

$$
\begin{aligned}
\Phi(A, B) & =\left(A \circ P_{B}^{b_{i}} \circ A^{-1}\right)_{1 \leq i \leq n}, \\
\Psi\left(\left(G_{i}\right)_{1 \leq i \leq n}\right) & =\left(\frac{1}{4}\left(D_{0}^{(3)}\left(G_{i} \circ G_{j}\right)-D_{0}^{(3)}\left(G_{j} \circ G_{i}\right)\right)\right)_{1 \leq i<j \leq n .}
\end{aligned}
$$

By $O_{\mathcal{M}_{2}}$, we denote the zero element of $\mathcal{M}_{2}=S^{3}\left(\mathbb{R}^{n}\right)^{n(n-1) / 2}$. Then,

$$
\Psi \circ \Phi(A, B)=O_{\mathcal{M}_{2}}, \quad \Psi\left(P^{b_{1}}, \ldots, P^{b_{n}}\right)=O_{\mathcal{M}_{2}}
$$

for any $(A, B) \in \mathcal{M}_{0}$ and $P \in \mathcal{M}_{1}^{\prime}$. Moreover, if $\Phi(A, B)=\left(P^{b_{1}}, \ldots, P^{b_{n}}\right)$, then $P$ is smoothly conjugate to $P_{B}$ by the linear map $A$.

The following is a direct corollary of Proposition 2.4.

Corollary 2.5 To prove Theorem 2.1, it is sufficient to show the existence of a $C_{\mathrm{loc}}^{2}-$ neighborhood $\mathcal{V}_{*}$ of $\left(P_{B}^{b_{i}}\right)_{1 \leq i \leq n}$ in $\mathcal{M}_{1}$ such that

$$
\Psi^{-1}\left(O_{\mathcal{M}_{2}}\right) \cap \mathcal{V}_{*}=\operatorname{Im} \Phi \cap \mathcal{V}_{*}
$$

for any given $B \in \mathrm{GL}_{n}(\mathbb{R})$. 
Let us recall Weil's Implicit Function Theorem.

Theorem 2.6 (Weil [18]) Let $\Phi_{0}: M_{0} \rightarrow M_{1}$ and $\Phi_{1}: M_{1} \rightarrow M_{2}$ be smooth maps between manifolds $M_{0}, M_{1}$, and $M_{2}$. Suppose that $\Phi_{1} \circ \Phi_{0}$ is a constant map with value $x_{2} \in M_{2}$. If $\operatorname{Ker}\left(D \Phi_{1}\right)_{x_{1}}=\operatorname{Im}\left(D \Phi_{0}\right)_{x_{0}}$ for $x_{0} \in M_{0}$ and $x_{1}=\Phi_{0}\left(x_{0}\right) \in M_{1}$, then there exists a neighborhood $U$ of $x_{1}$ such that $\operatorname{Im} \Phi_{0} \cap U=\Phi_{1}^{-1}\left(x_{2}\right) \cap U$.

The space $\mathcal{M}_{0}$ admits a natural smooth structure as an open subset of a finite dimensional vector space $M_{n}(\mathbb{R})^{2}$. The space $\mathcal{M}_{2}=\left(\mathcal{S}^{3}\left(\mathbb{R}^{n}\right)\right)^{n(n-1) / 2}$ also does as a finite dimensional vector space. If the maps $\Phi$ and $\Psi$ are smooth with respect to some smooth structure on $\mathcal{M}_{1}$ compatible to the $C_{\text {loc }}^{2}$-topology and satisfy $\operatorname{Ker} D \Psi_{\left(P_{B}^{b}, \ldots, P_{B}^{b n}\right)}=\operatorname{Im} D \Phi_{(I, B)}$, then Theorem 2.1 follows from Corollary 2.5 and Weil's theorem. To introduce a smooth structure on $\mathcal{M}_{1}$, we define a map $\Theta: \mathcal{M}_{1} \rightarrow \mathcal{S}^{2}\left(\mathbb{R}^{n}\right)^{n}$ by

$$
\Theta\left(G_{1}, \ldots, G_{n}\right)=\frac{1}{2}\left(D_{0}^{(2)} G_{1}, \ldots, D_{0}^{(2)} G_{n}\right) .
$$

Lemma 2.7 The map $\Theta$ is a homeomorphism with respect to the $C_{\text {loc }}^{2}$-topology on $\mathcal{M}_{1}$.

Proof Since $D_{0}^{(1)} G_{i}=I$ for any $\left(G_{1}, \ldots, G_{n}\right) \in \mathcal{M}_{1}$ and any $i$, the map $\Theta$ is continuous by the definition of the $C_{\text {loc }}^{2}$-topology.

Next, we show that $\Theta$ is surjective. Put $\left(e_{1}, \ldots, e_{n}\right)=I$ and take $Q \in \mathcal{S}^{2}\left(\mathbb{R}^{n}\right)$. Let $G_{Q}^{t} \in \mathcal{D}\left(\mathbb{R}^{n}, 0\right)$ be the time- $t$ map of the local flow generated by the quadratic vector field $X_{Q}(x)=Q(x, x)$. Then, $G_{Q}^{t}(0)=0, D_{0}^{(1)} G_{Q}^{t}=I$, and $\bar{F} \circ G_{Q}^{t}=G_{Q}^{k t} \circ \bar{F}$ for any $t \in \mathbb{R}$. Since

$$
\begin{aligned}
\left.\frac{d}{d t}\left[D_{0}^{(2)} G_{Q}^{t}\right]\left(e_{i}, e_{j}\right)\right|_{t=t_{0}} & =\left.\frac{\partial}{\partial t} \frac{\partial^{2}}{\partial x_{i} \partial x_{j}} G_{Q}^{t}(x)\right|_{(x, t)=\left(0, t_{0}\right)} \\
& =\left.\frac{\partial^{2}}{\partial x_{i} \partial x_{j}} \frac{\partial}{\partial t} G_{Q}^{t}(x)\right|_{(x, t)=\left(0, t_{0}\right)} \\
& =\left.\frac{\partial^{2}}{\partial x_{i} \partial x_{j}} Q\left(G_{Q}^{t_{0}}(x), G_{Q}^{t_{0}}(x)\right)\right|_{x=0} \\
& =2 Q\left(D_{0}^{(1)} G_{Q}^{t_{0}}\left(e_{i}\right), D_{0}^{(1)} G_{Q}^{t_{0}}\left(e_{j}\right)\right) \\
& =2 Q\left(e_{i}, e_{j}\right)
\end{aligned}
$$

for any $i, j=1, \ldots, n$ and $t_{0} \in \mathbb{R}$, we have $D_{0}^{(2)} G_{Q}^{t}=2 t Q$ for any $t$. Therefore, $\Theta\left(G_{Q_{1}}^{1}, \ldots, G_{Q_{n}}^{1}\right)=\left(Q_{1}, \ldots, Q_{n}\right)$ for any $\left(Q_{1}, \ldots, Q_{n}\right) \in \mathcal{S}^{2}\left(\mathbb{R}^{n}\right)^{n}$. 
Finally, we show $\Theta$ is injective. Note that the bijectivity of $\Theta$ implies that it is an open map. Take $G_{1}, G_{2} \in \mathcal{D}\left(\mathbb{R}^{N}, 0\right)$ such that $D_{0}^{(1)} G_{i}=I$ and $\bar{F} \circ G_{i}=G_{i}^{k} \circ \bar{F}$ for $i=1,2$, and $D_{0}^{(2)} G_{1}=D_{0}^{(2)} G_{2}$. We will show $G_{1}=G_{2}$. For $R>0$, we put $B_{R}=\left\{z \in \mathbb{R}^{N} \mid\|z\| \leq R\right\}$. Fix representatives $\widetilde{G}_{i}$ of $G_{i}$ for $i=1,2$. Since $G_{i}(0)=0$ and $D_{0}^{(1)} G_{i}=I$, there exists $R_{0}>0$ and $1<c<\sqrt[4]{k}$ such that

- $\widetilde{G}_{2}^{m} \circ \widetilde{G}_{1}^{m^{\prime}}$ is well-defined on $B_{R_{0}}$ for any $m, m^{\prime}=1, \ldots, k$,

- $\bar{F} \circ \widetilde{G}_{i}=\widetilde{G}_{i}^{k} \circ \bar{F}$ on $B_{R_{0}}$ for $i=1,2$,

- $\max \left\{\left\|\widetilde{G}_{1}^{m}(z)\right\|,\left\|\widetilde{G}_{2} \circ \widetilde{G}_{1}^{m}(z)\right\|\right\} \leq c\|z\|$, and $\left\|\widetilde{G}_{2}^{m}(z)-\widetilde{G}_{2}^{m}\left(z^{\prime}\right)\right\| \leq c\left\|z-z^{\prime}\right\|$ for any $z, z^{\prime} \in B_{R_{0}}$ and $m=1, \ldots, k$.

For $0<R \leq R_{0}$, we put

$$
\Delta(R)=\sup _{z \in B_{R}} \frac{\left\|\widetilde{G}_{1}(z)-\widetilde{G}_{2}(z)\right\|}{\|z\|^{3}} .
$$

Since $D_{0}^{(2)} G_{1}=D_{0}^{(2)} G_{2}$, then we have that $\widetilde{G}_{1}-\widetilde{G}_{2}$ is of at least third order at the origin. Hence, $\Delta(R)$ is finite. For any $z \in B_{R_{0}}$ and $m=1, \ldots, k$, we have $\max \left\{\left\|\widetilde{G}_{1}^{m}\left(k^{-1} z\right)\right\|,\left\|\widetilde{G}_{2} \circ \widetilde{G}_{1}^{m}\left(k^{-1} z\right)\right\|\right\} \leq(c / k)\|z\| \leq R_{0}$, and hence,

$$
\begin{aligned}
\left\|\widetilde{G}_{1}(z)-\widetilde{G}_{2}(z)\right\| & =k \cdot\left\|\bar{F} \circ \widetilde{G}_{1}(z)-\bar{F} \circ \widetilde{G}_{2}(z)\right\| \\
& =k \cdot\left\|\widetilde{G}_{1}^{k}\left(k^{-1} z\right)-\widetilde{G}_{2}^{k}\left(k^{-1} z\right)\right\| \\
& \leq k \sum_{m=1}^{k}\left\|\widetilde{G}_{2}^{m-1} \circ \widetilde{G}_{1}^{k-m+1}\left(k^{-1} z\right)-\widetilde{G}_{2}^{m} \circ \widetilde{G}_{1}^{k-m}\left(k^{-1} z\right)\right\| \\
& \leq k c \sum_{m=1}^{k}\left\|\widetilde{G}_{1}^{k-m+1}\left(k^{-1} z\right)-\widetilde{G}_{2} \circ \widetilde{G}_{1}^{k-m}\left(k^{-1} z\right)\right\| .
\end{aligned}
$$

Since $\left\|\widetilde{G}_{1}^{k-m}\left(k^{-1} z\right)\right\| \leq(c / k)\|z\| \leq R_{0}$, it implies that

$$
\left\|\widetilde{G}_{1}(z)-\widetilde{G}_{2}(z)\right\| \leq k^{2} c \cdot \Delta\left(R_{0}\right) \cdot[(c / k) \cdot\|z\|]^{3}=\left(c^{4} / k\right) \cdot \Delta\left(R_{0}\right) \cdot\|z\|^{3} .
$$

Therefore, $\Delta\left(R_{0}\right) \leq\left(c^{4} / k\right) \Delta\left(R_{0}\right)$. Since $c<\sqrt[4]{k}$, we have $\Delta\left(R_{0}\right)=0$, and hence, $\widetilde{G}_{1}=\widetilde{G}_{2}$ on $B_{R_{0}}$.

Since

$$
D_{0}^{(2)}\left(A \circ P_{B}^{b_{i}} \circ A^{-1}\right)=A \circ D_{0}^{(2)} P_{B}^{b_{i}} \circ\left(A^{-1}, A^{-1}\right)=2 A \circ Q_{v_{i}} \circ\left(A^{-1}, A^{-1}\right)
$$

for $A \in \mathrm{GL}_{n}(\mathbb{R})$ and $B=\left(v_{1}, \ldots, v_{n}\right) \in \mathrm{GL}_{n}(\mathbb{R})$, the map $\Theta \circ \Phi$ satisfies

$$
(\Theta \circ \Phi)(A, B)=\left(A \circ Q_{v_{i}} \circ\left(A^{-1}, A^{-1}\right)\right)_{1 \leq i \leq n} .
$$


Hence, $\Theta \circ \Phi$ is smooth. For $Q, Q^{\prime} \in \mathcal{S}^{2}\left(\mathbb{R}^{n}\right)$, we define $\left[Q, Q^{\prime}\right] \in \mathcal{S}^{3}\left(\mathbb{R}^{n}\right)$ by

$$
\begin{aligned}
{\left[Q, Q^{\prime}\right](\xi, \eta, \theta)=\left\{Q\left(\xi, Q^{\prime}(\eta, \theta)\right)+Q\left(\eta, Q^{\prime}(\theta, \xi)\right)+Q\left(\theta, Q^{\prime}(\xi, \eta)\right)\right\} } \\
-\left\{Q^{\prime}(\xi, Q(\eta, \theta))+Q^{\prime}(\eta, Q(\theta, \xi))+Q^{\prime}(\theta, Q(\xi, \eta))\right\}
\end{aligned}
$$

It can be checked that

$$
\left[D_{0}^{(2)} G_{1}, D_{0}^{(2)} G_{2}\right]=D_{0}^{(3)}\left(G_{1} \circ G_{2}\right)-D_{0}^{(3)}\left(G_{2} \circ G_{1}\right)
$$

for any $G_{1}, G_{2} \in \mathcal{D}\left(\mathbb{R}^{N}, 0\right)$ with $D_{0}^{(1)} G_{1}=D_{0}^{(1)} G_{2}=I$. Therefore,

$$
\left(\Psi \circ \Theta^{-1}\right)\left(Q_{1}, \ldots, Q_{n}\right)=\left(\left[Q_{i}, Q_{j}\right]\right)_{1 \leq i<j \leq n} .
$$

Since the bracket is bilinear, the map $\Psi \circ \Theta^{-1}$ is a smooth map.

For $B=\left(v_{1}, \ldots, v_{n}\right) \in \mathrm{GL}_{n}(\mathbb{R})$, we put

$$
\begin{aligned}
& L_{B}^{\Phi}=D(\Theta \circ \Phi)_{(I, B)}, \\
& L_{B}^{\Psi}=D\left(\Psi \circ \Theta^{-1}\right)_{\left(Q_{v_{1}}, \ldots, Q_{v_{n}}\right) .}
\end{aligned}
$$

We identify the tangent spaces of $\mathcal{M}_{0}$ and $\mathcal{S}^{2}\left(\mathbb{R}^{n}\right)^{n}$ of each point with $M_{n}\left(\mathbb{R}^{n}\right)^{2}$ and $\mathcal{S}^{2}\left(\mathbb{R}^{n}\right)^{n}$, respectively. Then, (2) and (4) imply that

$$
\begin{aligned}
L_{B}^{\Phi}\left(A^{\prime}, B^{\prime}\right) & =\left(A^{\prime} \circ Q_{v_{i}}-Q_{v_{i}} \circ\left(A^{\prime}, I\right)-Q_{v_{i}} \circ\left(I, A^{\prime}\right)+Q_{\omega_{i}}\right)_{1 \leq i \leq n}, \\
L_{B}^{\Psi}\left(q_{1}, \ldots, q_{n}\right) & =\left(\left[q_{i}, Q_{v_{j}}\right]-\left[q_{j}, Q_{v_{i}}\right]\right)_{1 \leq i<j \leq n}
\end{aligned}
$$

for any $\left(A^{\prime}, B^{\prime}\right) \in M_{n}(\mathbb{R})^{2}$ with $B^{\prime}=\left(\omega_{1}, \ldots, \omega_{n}\right)$ and any $\left(q_{1}, \ldots, q_{n}\right) \in \mathcal{S}^{2}\left(\mathbb{R}^{n}\right)^{n}$. The following proposition can be shown by a formal computation and we postpone the proof until Section 2.3.

Proposition 2.8 $\operatorname{Ker} L_{B}^{\Psi}=\operatorname{Im} L_{B}^{\Phi}$.

Theorem 2.1 follows from Corollary 2.5, Theorem 2.6, and the proposition since $H$ is a homeomorphism between $\mathcal{M}_{1}$ and $\mathcal{S}^{2}\left(\mathbb{R}^{n}\right)^{n}$.

\subsection{From local to global}

In this subsection, we prove the main theorem. For a discrete group $\Gamma$ and a $\Gamma$-action $\rho$ on a manifold $M$, we say a point $p \in M$ is a global fixed point if $\rho^{\gamma}(p)=p$ for any $\gamma \in \Gamma$. Note the point $\infty$ is the unique global fixed point of $\rho_{B}$ for any $B \in \mathrm{GL}_{n}(\mathbb{R})$.

In this subsection, we assume that $n \geq 2$ since the case $n=1$ was already shown by Burslem and Wilkinson. First, we show that any local conjugacy to the standard $\Gamma_{n, k}$-action extends to a global one. 
Proposition 2.9 Suppose that an action $\rho \in \operatorname{Hom}\left(\Gamma_{n, k}, \operatorname{Diff}(M)\right)$ admits a global fixed point $p_{\infty}$ and there exists a smooth coordinate $\phi$ of $S^{n}$ at $p_{\infty}$ and $B \in \mathrm{GL}_{n}(\mathbb{R})$ such that $\phi\left(p_{\infty}\right)=0$ and $\phi \circ \rho^{\gamma} \circ \phi^{-1}=P_{B}^{\gamma}$ as elements of $\mathcal{D}\left(\mathbb{R}^{n}, 0\right)$ for any $\gamma \in \Gamma_{n, k}$. Then, $\rho$ is smoothly conjugate to $\rho_{B}$.

Proof Recall $\bar{\phi}: S^{n} \rightarrow \mathbb{R}^{n}$ is the local coordinate at $\infty$ given by $\bar{\phi}(x)=\left(1 /\|x\|^{2}\right) \cdot x$ and the local action $P_{B}$ is defined by $P_{B}^{\gamma}=\bar{\phi} \circ \rho_{B}^{\gamma} \circ \bar{\phi}$. We put $U_{r}=S^{n} \backslash[-r, r]^{n}$ for $r>0$ and $\Lambda_{b}=\left\{b_{1}^{ \pm 1}, \ldots, b_{n}^{ \pm 1}\right\}$. By assumption, there exists $R>0$ and a neighborhood $U^{\prime}$ of $p_{\infty}$ such that

$$
\phi \circ \rho^{\gamma} \circ \phi^{-1}=\bar{\phi} \circ \rho_{B}^{\gamma} \circ \bar{\phi}^{-1}
$$

on $\bar{\phi}\left(U_{R}\right)$ for any $\gamma \in\left\{a^{ \pm 1}\right\} \cup \Lambda_{b}$. Since $\rho_{B}^{b_{1}^{m}}(x)$ converges to $\infty$ as $n$ goes to infinity for any $x \in S^{n}$, we can take $m_{x} \geq 0$ such that $\rho_{B}^{b_{1} m_{x}}(x)$ is contained in $U_{R}$. Define a map $h: S^{n} \rightarrow S^{n}$ by

$$
h(x)=\rho^{b_{1}^{-m_{x}}} \circ\left(\phi^{-1} \circ \bar{\phi}\right) \circ \rho_{B}^{b_{1}^{m_{x}}}(x) .
$$

First, we see $h(x)$ does not depend on the choice of $m_{x}$. Suppose $\rho_{B}^{b_{1}^{m}}(x)$ is contained in $U_{R}$. Since $\rho_{B}^{\gamma}$ is a translation for any $\gamma \in \Lambda_{b}$ and $S^{n} \backslash U_{R}=[-R, R]^{n}$ is a convex subset of $\mathbb{R}^{n}$, there exists a sequence $\left(\gamma_{j}\right)_{1 \leq j \leq l}$ of elements of $\Lambda_{b}$ so that $b_{1}^{m}=\gamma_{l} \cdots \gamma_{1} b_{1}^{m_{x}}$ and $\rho_{B}^{\gamma_{j} \cdots \gamma_{1} b_{1}^{m_{x}}}(x)$ is contained in $U_{R}$ for any $j=1, \ldots, l{ }^{1}$ Then,

$$
\rho^{\gamma_{j+1}} \circ\left(\phi^{-1} \circ \bar{\phi}\right) \circ \rho_{B}^{\gamma_{j} \cdots \gamma_{1} b_{1}^{m_{x}}}(x)=\left(\phi^{-1} \circ \bar{\phi}\right) \circ \rho_{B}^{\gamma_{j+1} \gamma_{j} \cdots \gamma_{1} b_{1}^{m_{x}}}(x) \text {. }
$$

This implies that

$$
\begin{aligned}
& \rho^{b_{1}^{-m}} \circ\left(\phi^{-1} \circ \bar{\phi}\right) \circ \rho^{b_{1}^{m}}(x)=\rho^{b_{1}^{-m}} \circ\left(\phi^{-1} \circ \bar{\phi}\right) \circ \rho_{B}^{\gamma_{l} \cdots \gamma_{1}} \circ \rho^{b_{1}^{m_{x}}}(x) \\
& =\rho^{b_{1}^{-m}} \circ \rho^{\gamma_{l} \cdots \gamma_{1}} \circ\left(\phi^{-1} \circ \bar{\phi}\right) \circ \rho^{b_{1}^{m_{x}}}(x)
\end{aligned}
$$

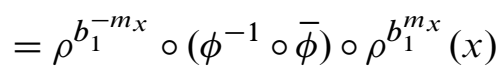

$$
\begin{aligned}
& =h(x) \text {. }
\end{aligned}
$$

Therefore, $h(x)$ does not depend on the choice of $m_{x}$.

For any given $x_{0} \in S^{n}$, there is a choice of $\left(m_{x}\right)_{x \in S^{n}}$ which is constant on a small neighborhood of $x_{0}$. This implies that $h$ is a locally diffeomorphic at $x_{0}$, and hence, $h$ is a covering map. Since $S^{n}$ is simply connected, $h$ is diffeomorphism.

${ }^{1}$ We need $n \geq 2$ here. 
It is easy to see $h \circ \rho_{B}^{\gamma}=\rho^{\gamma} \circ h$ for any $\gamma \in \Lambda_{b}$. For any given $x \in S^{n}$, there is $m \geq 1$ such that $\rho_{B}^{b_{1}^{k m}}(x)$ is contained in $U_{R}$. Then,

$$
\begin{aligned}
h \circ \rho_{B}^{a}(x) & =\rho^{b_{1}^{-k m}} \circ\left(\phi^{-1} \circ \bar{\phi}\right) \circ \rho_{B}^{b_{1}^{k m}} \circ \rho_{B}^{a}(x) \\
& =\rho b_{1}^{-k m} \circ\left(\phi^{-1} \circ \bar{\phi}\right) \circ \rho_{B}^{a} \circ \rho_{B}^{b_{1}^{m}}(x) \\
& =\rho b_{1}^{-k m} \circ \rho^{a} \circ\left(\phi^{-1} \circ \bar{\phi}\right) \circ \rho_{B}^{b_{1}^{m}}(x) \\
& =\rho^{a} \circ \rho b_{1}^{-m} \circ\left(\phi^{-1} \circ \bar{\phi}\right) \circ \rho_{B}^{b_{1}^{m}}(x) \\
& =\rho^{a} \circ h(x) .
\end{aligned}
$$

Therefore, $h$ is a smooth conjugacy between $\rho_{B}$ and $\rho$.

Next, we give a criterion for the persistence of a global fixed point of a $\Gamma_{n, k}-$ action.

Lemma 2.10 Let $M$ be a manifold and $\rho$ be an action in $\operatorname{Hom}\left(\Gamma_{n, k}, \operatorname{Diff}(M)\right)$. Suppose that $\rho_{0}$ has a global fixed point $p_{0}$ such that $\left(D \rho_{0}^{a}\right)_{p_{0}}=k^{-1} I$ and $\left(D \rho_{0}^{b_{i}}\right)_{p_{0}}=I$ for any $i=1, \ldots, n$. Then, there exists a neighborhood $\mathcal{U} \subset \operatorname{Hom}\left(\Gamma_{n, k}, \operatorname{Diff}(M)\right)$ of $\rho_{0}$ and a continuous map $\hat{p}: \mathcal{U} \rightarrow M$ such that $\hat{p}\left(\rho_{0}\right)=p_{0}$ and $\hat{p}(\rho)$ is a global fixed point of $\rho$ for any $\rho \in \mathcal{U}$.

Proof Take $k^{-1}<\lambda<1$ and $\delta>0$ so that $\lambda+k \delta<1$. Fix an open neighborhood $U$ of $p_{0}$ and a local coordinate $\phi: U \rightarrow \mathbb{R}^{n}$. There exist convex neighborhoods $V$ and $V_{1}$ of $\phi\left(p_{0}\right)$ and a neighborhood $\mathcal{U}_{0}$ of $\rho_{0}$ which satisfy the following conditions for any $\rho \in \mathcal{U}_{0}$ and $i=1, \ldots, n$.

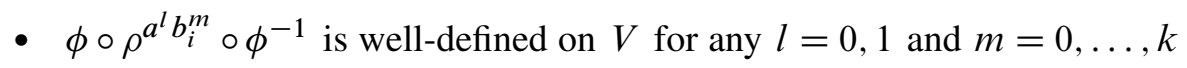

- $\phi \circ \rho^{b_{i}} \circ \phi^{-1}\left(V_{1}\right) \subset V$

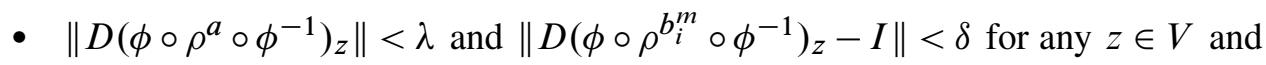
$m=1, \ldots, k$

By the persistence of attracting fixed point, there exists a neighborhood $\mathcal{U} \subset \mathcal{U}_{0}$ of $\rho_{0}$ and a continuous map $\hat{p}: \mathcal{U} \rightarrow \phi^{-1}\left(V_{1} \cap V\right)$ such that $\hat{p}\left(\rho_{0}\right)=p_{0}$ and $\hat{p}(\rho)$ is an attracting fixed point of $\rho^{a}$ for any $\rho \in \mathcal{U}$. Since $\rho_{0}^{b_{i}}\left(p_{0}\right)=p_{0}$, by replacing $\mathcal{U}$ with a smaller neighborhood of $\rho_{0}$, we may assume $\rho^{b_{i}}(\hat{p}(\rho)) \in \phi^{-1}\left(V_{1} \cap V\right)$ for any $\rho \in \mathcal{U}$ and $i=1, \ldots, n$.

Fix $i=1, \ldots, n$ and $\rho \in \mathcal{U}$. Put $z_{*}=\phi(\hat{p}(\rho)), F=\phi \circ \rho^{a} \circ \phi^{-1}$, and $G=\phi \circ \rho^{b_{i}} \circ \phi^{-1}$. We will show $G\left(z_{*}\right)=z_{*}$. Since $z_{*}$ and $G\left(z_{*}\right)$ are contained in $V$,

$$
\begin{gathered}
\left\|F \circ G\left(z_{*}\right)-F\left(z_{*}\right)\right\| \leq \lambda\left\|G\left(z_{*}\right)-z_{*}\right\|, \\
\left\|\left(G^{m+1}\left(z_{*}\right)-G\left(z_{*}\right)\right)-\left(G^{m}\left(z_{*}\right)-z_{*}\right)\right\| \leq \delta\left\|G\left(z_{*}\right)-z_{*}\right\|,
\end{gathered}
$$


for $m=0, \ldots, k-1$. Since $F \circ G=G^{k} \circ F$ and $F\left(z_{*}\right)=z_{*}$, the former implies

$$
\left\|G^{k}\left(z_{*}\right)-z_{*}\right\| \leq \lambda\left\|G\left(z_{*}\right)-z_{*}\right\| .
$$

Hence,

$$
\begin{aligned}
k \cdot\left\|z_{*}-G\left(z_{*}\right)\right\| & \leq\left\|G^{k}\left(z_{*}\right)-z_{*}\right\|+\sum_{m=0}^{k-1}\left\|G^{m+1}\left(z_{*}\right)-G^{m}\left(z_{*}\right)-G\left(z_{*}\right)+z_{*}\right\| \\
& \leq(\lambda+k \delta) \cdot\left\|G\left(z_{*}\right)-z_{*}\right\| .
\end{aligned}
$$

Since $\lambda+k \delta<1$, we have $G\left(z_{*}\right)=z_{*}$. Thus, $\hat{p}(\rho)$ is a global fixed point of $\rho$.

Now, we prove the main theorem.

Proof of Main Theorem Take open neighborhoods $U \subset S^{n}$ of $\infty$ and $V \subset \mathbb{R}^{n}$ of 0 and a family $\left(\phi_{p}\right)_{p \in U}$ of diffeomorphisms from $U$ to $V$ such that $\phi_{\infty}=\bar{\phi}, \phi_{p}(p)=0$ for any $p \in U$ and the map $(p, q) \mapsto \phi_{p}(q)$ is smooth. Fix $B \in \mathrm{GL}_{n}(\mathbb{R})$. The action $\rho_{B}$ satisfies the assumption of Lemma 2.10. Hence, there exists a neighborhood $\mathcal{U}_{1}$ of $\rho_{B}$ and a continuous map $\hat{p}: \mathcal{U}_{1} \rightarrow U$ such that $\hat{p}(\rho)$ is a global fixed point of $\rho$ for any $\rho \in \mathcal{U}_{1}$. We define a local action $P_{\rho} \in \operatorname{Hom}\left(\Gamma_{n, k}, \mathcal{D}\left(\mathbb{R}^{n}, 0\right)\right)$ by $P_{\rho}^{\gamma}=\phi_{\hat{p}(\rho)} \circ \rho^{\gamma} \circ \phi_{\hat{p}(\rho)}^{-1}$. Then, the map $\rho \mapsto P_{\rho}$ is a $C_{\text {loc }}^{2}$-continuous map from $\mathcal{U}_{1}$ to $\operatorname{Hom}\left(\Gamma_{n, k}, \mathcal{D}\left(\mathbb{R}^{n}, 0\right)\right)$. By Theorem 2.1, there exists a neighborhood $\mathcal{U} \subset \mathcal{U}_{1}$ of $\rho_{B}$ such that $P_{\rho}$ is smoothly conjugate to $P_{B^{\prime}}$ for some $B^{\prime}=B^{\prime}(\rho) \in \mathrm{GL}_{n}(\mathbb{R})$ for any $\rho \in \mathcal{U}$. By Proposition 2.9, $\rho$ is smoothly conjugate to $\rho_{B^{\prime}}$.

\subsection{Proof of Proposition 2.8}

In this subsection, we give a proof of the following proposition, which we have not shown in Section 2.1 .

\section{Proposition 2.8 $\operatorname{Ker} L_{B}^{\Psi}=\operatorname{Im} L_{B}^{\Phi}$.}

Our proof is formal and lengthy computation. It may be interesting to find a more geometric proof.

Fix $B=\left(v_{1}, \ldots, v_{n}\right) \in \mathrm{GL}_{n}(\mathbb{R})$. Recall the linear maps $L_{B}^{\Phi}: M_{n}\left(\mathbb{R}^{n}\right)^{2} \rightarrow \mathcal{S}^{2}\left(\mathbb{R}^{n}\right)^{n}$ and $L_{B}^{\Psi}: \mathcal{S}^{2}\left(\mathbb{R}^{n}\right)^{n} \rightarrow \mathcal{S}^{3}\left(\mathbb{R}^{n}\right)^{n(n-1) / 2}$ are given by

$$
\begin{aligned}
L_{B}^{\Phi}\left(A^{\prime}, B^{\prime}\right) & =\left(A^{\prime} \circ Q_{v_{i}}-Q_{v_{i}} \circ\left(A^{\prime}, I\right)-Q_{v_{i}} \circ\left(I, A^{\prime}\right)+Q_{\omega_{i}}\right)_{1 \leq i \leq n}, \\
L_{B}^{\Psi}\left(q_{1}, \ldots, q_{n}\right) & =\left(\left[q_{i}, Q_{v_{j}}\right]-\left[q_{j}, Q_{v_{i}}\right]\right)_{1 \leq i<j \leq n}
\end{aligned}
$$


for any $\left(A^{\prime}, B^{\prime}\right) \in M_{n}(\mathbb{R})^{2}$ with $B^{\prime}=\left(\omega_{1}, \ldots, \omega_{n}\right)$ and any $\left(q_{1}, \ldots, q_{n}\right) \in \mathcal{S}^{2}\left(\mathbb{R}^{n}\right)^{n}$, where

$$
\begin{aligned}
Q_{v}(\xi, \eta) & =\langle\xi, \eta\rangle \cdot v-\langle\xi, v\rangle \cdot \eta-\langle\eta, v\rangle \cdot \xi \\
{\left[Q, Q^{\prime}\right](\xi, \eta, \theta) } & =\left\{Q\left(\xi, Q^{\prime}(\eta, \theta)\right)+Q\left(\eta, Q^{\prime}(\theta, \xi)\right)+Q\left(\theta, Q^{\prime}(\xi, \eta)\right)\right\} \\
& \quad-\left\{Q^{\prime}(\xi, Q(\eta, \theta))+Q^{\prime}(\eta, Q(\theta, \xi))+Q^{\prime}(\theta, Q(\xi, \eta))\right\} .
\end{aligned}
$$

First, we reduce the problem to the case $B=I$.

Lemma 2.11 For $B, B^{\prime} \in \mathrm{GL}_{n}(\mathbb{R}), \operatorname{Ker} L_{B}^{\Psi}=\operatorname{Im} L_{B}^{\Phi}$ if and only if $\operatorname{Ker} L_{B^{\prime}}^{\Psi}=\operatorname{Im} L_{B^{\prime}}^{\Phi}$.

Proof Put $B=\left(v_{1}, \ldots, v_{n}\right)$ and $B^{\prime}=\left(w_{1}, \ldots, w_{n}\right)$. Take $A=\left(a_{i j}\right) \in \mathrm{GL}_{n}(\mathbb{R})$ such that $B^{\prime}=B A$. Since the map $v \mapsto Q_{v}$ is linear, we have

$$
\left(Q_{w_{1}}, \ldots, Q_{w_{n}}\right)=\left(Q_{v_{1}}, \ldots, Q_{v_{n}}\right) \cdot A .
$$

It implies that $\operatorname{Im} L_{B^{\prime}}^{\Phi}=\operatorname{Im} L_{B}^{\Phi} \cdot A$. For $\left(q_{1}, \ldots, q_{n}\right) \in \operatorname{Ker} L_{B}^{\Psi}$ we have

$\left[\left(\sum_{k=1}^{n} a_{k i} q_{k}\right), Q_{w_{j}}\right]-\left[\left(\sum_{l=1}^{n} a_{l j} q_{l}\right), Q_{w_{i}}\right]=\sum_{k, l=1}^{n} a_{k i} a_{l j}\left(\left[q_{k}, Q_{v_{l}}\right]-\left[q_{l}, Q_{v_{k}}\right]\right)=0$.

Hence, $\operatorname{Ker} L_{B}^{\Psi} \cdot A$ is a subspace of $\operatorname{Ker} L_{B^{\prime}}^{\Psi}$. Similarly, $\operatorname{Ker} L_{B^{\prime}}^{\Psi} \cdot A^{-1}$ is a subspace of $\operatorname{Ker} L_{B}^{\Psi}$. Thus, $\operatorname{Ker} L_{B^{\prime}}^{\Psi}=\operatorname{Ker} L_{B}^{\Psi} \cdot A$.

By the lemma, it is sufficient to show Proposition 2.8 for $B=I$. Put $I=\left(e_{1}, \ldots, e_{n}\right)$. It is easy to check the following properties of $Q_{v}$.

Lemma 2.12 For $v \in \mathbb{R}^{n}$ and mutually disjoint $i, j, k=1, \ldots, n$,

$$
\begin{gathered}
Q_{e_{i}}\left(e_{i}, v\right)=Q_{e_{i}}\left(v, e_{i}\right)=-v, \\
Q_{e_{i}}\left(e_{j}, e_{j}\right)=e_{i}, \\
Q_{e_{i}}\left(e_{j}, e_{k}\right)=0 .
\end{gathered}
$$

Let $W$ be the subspace of $\mathcal{S}^{2}\left(\mathbb{R}^{n}\right)^{n}$ consisting of $\left(q_{1}, \ldots, q_{n}\right)$ such that

$$
\begin{gathered}
q_{j}\left(e_{j}, e_{j}\right)=0, \\
\left\langle e_{i}, q_{j}\left(e_{i}, e_{i}\right)\right\rangle+\left\langle e_{j}, q_{i}\left(e_{j}, e_{j}\right)\right\rangle=0, \\
\left\langle e_{1}, q_{1}\left(e_{j}, e_{j}\right)\right\rangle=0,
\end{gathered}
$$

for any $i, j=1, \ldots, n$. 
Lemma 2.13 If $\operatorname{Ker} L_{I}^{\Psi} \cap W=\{0\}$, then $\operatorname{Ker} L_{I}^{\Psi}=\operatorname{Im} L_{I}^{\Phi}$.

Proof We show $\mathcal{S}^{2}\left(\mathbb{R}^{n}\right)^{n}=W+\operatorname{Im} L_{I}^{\Phi}$. Then the assumption $\operatorname{Ker} L_{I}^{\Psi} \cap W=\{0\}$ implies $\operatorname{Ker} L_{I}^{\Psi}=\operatorname{Im} L_{I}^{\Phi}$ since $\operatorname{Im} L_{I}^{\Phi} \subset \operatorname{Ker} L_{I}^{\Psi}$.

For $A, B \in M_{n}(\mathbb{R})$, let $q_{j}^{A, B}$ be the $j$-th component of $L_{I}^{\Phi}(A, B)$. Fix $\left(q_{1}, \ldots, q_{n}\right)$ in $\mathcal{S}^{2}\left(\mathbb{R}^{n}\right)^{n}$ and we will find $A, B \in M_{n}(\mathbb{R})$ such that

$$
q_{j}^{A, B}\left(e_{j}, e_{j}\right)=q_{j}\left(e_{j}, e_{j}\right),
$$

$$
\begin{aligned}
\left\langle e_{i}, q_{j}^{A, B}\left(e_{i}, e_{i}\right)\right\rangle+\left\langle e_{j}, q_{i}^{A, B}\left(e_{j}, e_{j}\right)\right\rangle & =\left\langle e_{i}, q_{j}\left(e_{i}, e_{i}\right)\right\rangle+\left\langle e_{j}, q_{i}\left(e_{j}, e_{j}\right)\right\rangle, \\
\left\langle e_{1}, q_{1}^{A, B}\left(e_{j}, e_{j}\right)\right\rangle & =\left\langle e_{1}, q_{1}\left(e_{j}, e_{j}\right)\right\rangle .
\end{aligned}
$$

These equations imply that $\left(q_{1}, \ldots, q_{n}\right)-L_{I}^{\Phi}(A, B)$ is an element of $W$.

Take $A=\left(a_{i j}\right), B=\left(b_{i j}\right) \in M_{n}(\mathbb{R})$. A direct computation with Lemma 2.12 implies

$$
\begin{aligned}
q_{j, B}^{\left.A, e_{j}, e_{j}\right)} & =A \circ Q_{e_{j}}\left(e_{j}, e_{j}\right)-2 Q_{e_{j}}\left(A e_{j}, e_{j}\right)+Q_{B e_{j}}\left(e_{j}, e_{j}\right) \\
& =A e_{j}+\sum_{k=1}^{n} b_{k j} Q_{e_{k}}\left(e_{j}, e_{j}\right) \\
& =\left(a_{j j}-b_{j j}\right) e_{j}+\sum_{k \neq j}\left(a_{k j}+b_{k j}\right) e_{k},
\end{aligned}
$$

$$
q_{i}^{A, B}\left(e_{j}, e_{j}\right)=A \circ Q_{e_{i}}\left(e_{j}, e_{j}\right)-2 Q_{e_{i}}\left(A e_{j}, e_{j}\right)+Q_{B e_{i}}\left(e_{j}, e_{j}\right)
$$

$$
\begin{aligned}
& =A e_{i}-2 \sum_{k=1}^{n} a_{k j} Q_{e_{i}}\left(e_{k}, e_{j}\right)+\sum_{k=1}^{n} b_{k i} Q_{e_{k}}\left(e_{j}, e_{j}\right) \\
& =\left(a_{i i}-2 a_{j j}+b_{i i}\right) e_{i}+\left(a_{j i}+2 a_{i j}-b_{j i}\right) e_{j}+\sum_{k \neq i, j}\left(a_{k i}+b_{k i}\right) e_{k} .
\end{aligned}
$$

for any mutually distinct $i, j=1, \ldots, n$. The latter equation implies that

$$
\left\langle e_{i}, q_{j}^{A, B}\left(e_{i}, e_{i}\right)\right\rangle+\left\langle e_{j}, q_{i}^{A, B}\left(e_{j}, e_{j}\right)\right\rangle=3\left(a_{i j}+a_{j i}\right)-\left(b_{i j}+b_{j i}\right)
$$

for any mutually distinct $i, j=1, \ldots, n$ and

$$
\left\langle e_{1}, q_{1}^{A, B}\left(e_{j}, e_{j}\right)\right\rangle=a_{11}-2 a_{j j}+b_{11}
$$

for any $j=2, \ldots, n$.

Put $s_{i j}=\left\langle e_{i}, q_{j}\left(e_{j}, e_{j}\right)\right\rangle, t_{i j}=\left\langle e_{j}, q_{i}\left(e_{j}, e_{j}\right)\right\rangle$ and $u_{j}=\left\langle e_{1}, q_{1}\left(e_{j}, e_{j}\right)\right\rangle$ for $i, j=$ $1, \ldots, n$. Notice that $s_{11}=t_{11}=u_{1}$. Put $a_{11}=s_{11} / 2, b_{11}=-s_{11} / 2$,

$$
a_{j j}=-u_{j} / 2, \quad b_{j j}=-s_{j j}-\left(u_{j} / 2\right)
$$


for $j=2, \ldots, n$, and

$$
a_{i j}=\frac{1}{4}\left(s_{i j}+t_{i j}\right), \quad b_{i j}=s_{i j}-a_{i j}=\frac{1}{4}\left(3 s_{i j}-t_{i j}\right),
$$

for any mutually distinct $i, j=1, \ldots, n$. By (12), (13), and (14), $A=\left(a_{i j}\right)$ and $B=\left(b_{i j}\right)$ satisfy $(9),(10)$, and (11).

Fix $\left(q_{1}, \ldots, q_{n}\right) \in \operatorname{Ker} L_{I}^{\Psi} \cap W$. By the lemma, the goal is to show $q_{1}=\cdots=q_{n}=0$.

Lemma $2.14 q_{j}\left(e_{i}, e_{j}\right)=q_{j}\left(e_{j}, e_{i}\right)=0$ for any $i, j=1, \ldots, n$.

Proof When $i=j$, it is just shown by (6) in the definition of $W$. Take mutually distinct $i, j=1, \ldots, n$. Then,

$$
\begin{aligned}
0 & =\frac{1}{3}\left(\left[q_{i}, Q_{e_{j}}\right]-\left[q_{j}, Q_{e_{i}}\right]\right)\left(e_{j}, e_{j}, e_{j}\right) \\
& =\left\{q_{i}\left(e_{j}, Q_{e_{j}}\left(e_{j}, e_{j}\right)\right)-Q_{e_{j}}\left(e_{j}, q_{i}\left(e_{j}, e_{j}\right)\right)\right\} \\
& \quad-\left\{q_{j}\left(e_{j}, Q_{e_{i}}\left(e_{j}, e_{j}\right)\right)-Q_{e_{i}}\left(e_{j}, q_{j}\left(e_{j}, e_{j}\right)\right)\right\} \\
& =\left\{q_{i}\left(e_{j},-e_{j}\right)+q_{i}\left(e_{j}, e_{j}\right)\right\}-\left\{q_{j}\left(e_{j}, e_{i}\right)-Q_{e_{i}}\left(e_{j}, 0\right)\right\} \\
& =-q_{j}\left(e_{j}, e_{i}\right) .
\end{aligned}
$$

Since $q_{j}$ is symmetric, we also obtain that $q_{j}\left(e_{i}, e_{j}\right)=0$.

Lemma 2.15 For any $i, j=1, \ldots, n$,

$$
\left\langle e_{i}, q_{i}\left(e_{j}, e_{j}\right)\right\rangle+\left\langle e_{j}, q_{j}\left(e_{i}, e_{i}\right)\right\rangle=0 .
$$

For any $i, j, k=1, \ldots, n$ with $i \neq k$,

$$
\left\langle e_{k}, q_{i}\left(e_{j}, e_{j}\right)\right\rangle=0 .
$$

Proof When $i=j$, Lemma 2.15 follows from the definition of $W$. Suppose $i \neq j$. Since $q_{i}\left(e_{i}, e_{j}\right)=q_{j}\left(e_{i}, e_{j}\right)=q_{j}\left(e_{j}, e_{j}\right)=0$ by Lemma 2.14 and (6) in the definition of $W$, we have

$$
\begin{aligned}
{\left[q_{i}, Q_{e_{j}}\right]\left(e_{i}, e_{j}, e_{j}\right)=} & \left\{q_{i}\left(e_{i}, Q_{e_{j}}\left(e_{j}, e_{j}\right)\right)+2 q_{i}\left(e_{j}, Q_{e_{j}}\left(e_{i}, e_{j}\right)\right)\right\} \\
& -\left\{Q_{e_{j}}\left(e_{i}, q_{i}\left(e_{j}, e_{j}\right)\right)+2 Q_{e_{j}}\left(e_{j}, q_{i}\left(e_{i}, e_{j}\right)\right)\right\} \\
= & \left\{q_{i}\left(e_{i},-e_{j}\right)+2 q_{i}\left(e_{j},-e_{i}\right)\right\} \\
& \quad-\left\{\left\langle e_{i}, q_{i}\left(e_{j}, e_{j}\right)\right\rangle \cdot e_{j}-\left\langle e_{j}, q_{i}\left(e_{j}, e_{j}\right)\right\rangle \cdot e_{i}+2 Q_{e_{j}}\left(e_{j}, 0\right)\right\} \\
= & \left\langle e_{j}, q_{i}\left(e_{j}, e_{j}\right)\right\rangle \cdot e_{i}-\left\langle e_{i}, q_{i}\left(e_{j}, e_{j}\right)\right\rangle \cdot e_{j},
\end{aligned}
$$




$$
\begin{aligned}
{\left[q_{j}, Q_{e_{i}}\right]\left(e_{i}, e_{j}, e_{j}\right)=} & \left\{q_{j}\left(e_{i}, Q_{e_{i}}\left(e_{j}, e_{j}\right)\right)+2 q_{j}\left(e_{j}, Q_{e_{i}}\left(e_{i}, e_{j}\right)\right)\right\} \\
& \quad-\left\{Q_{e_{i}}\left(e_{i}, q_{j}\left(e_{j}, e_{j}\right)\right)+2 Q_{e_{i}}\left(e_{j}, q_{j}\left(e_{i}, e_{j}\right)\right)\right\} \\
= & \left\{q_{j}\left(e_{i}, e_{i}\right)+2 q_{j}\left(e_{j},-e_{j}\right)\right\}-\left\{Q_{e_{i}}\left(e_{i}, 0\right)+2 Q_{e_{i}}\left(e_{j}, 0\right)\right\} \\
= & q_{j}\left(e_{i}, e_{i}\right) .
\end{aligned}
$$

Since $\left[q_{i}, Q_{e_{j}}\right]-\left[q_{j}, Q_{e_{j}}\right]=0$,

$$
q_{j}\left(e_{i}, e_{i}\right)=\left\langle e_{j}, q_{i}\left(e_{j}, e_{j}\right)\right\rangle \cdot e_{i}-\left\langle e_{i}, q_{i}\left(e_{j}, e_{j}\right)\right\rangle \cdot e_{j} .
$$

By taking the inner product with $e_{k}$, we obtain $\left\langle q_{i}\left(e_{j}, e_{j}\right), e_{k}\right\rangle=0$ for $k \neq i, j$. By taking the inner product with $e_{i}$ and $e_{j}$, we also have

$$
\begin{aligned}
& \left\langle e_{i}, q_{j}\left(e_{i}, e_{i}\right)\right\rangle-\left\langle e_{j}, q_{i}\left(e_{j}, e_{j}\right)\right\rangle=0, \\
& \left\langle e_{j}, q_{j}\left(e_{i}, e_{i}\right)\right\rangle+\left\langle e_{i}, q_{i}\left(e_{j}, e_{j}\right)\right\rangle=0 .
\end{aligned}
$$

The latter is (15). Equation (16) follows from the former and (7) in the definition of $W$.

Equations (8) and (15) imply that

$$
\left\langle e_{1}, q_{1}\left(e_{j}, e_{j}\right)\right\rangle=\left\langle e_{j}, q_{j}\left(e_{1}, e_{1}\right)\right\rangle=0
$$

for any $j=1, \ldots, n$. Now, we prove Proposition 2.8 for $n=2$.

Proposition 2.16 If $n=2$, then $\operatorname{Ker} L_{I}^{\Psi}=\operatorname{Im} L_{I}^{\Phi}$.

Proof For $\left(q_{1}, q_{2}\right) \in \operatorname{Ker} L_{I}^{\Psi} \cap W,\left\langle e_{i}, q_{j}\left(e_{k}, e_{l}\right)\right\rangle=0$ for any $i, j, k, l=1,2$ by Lemma 2.14, Lemma 2.15 and (17). Therefore, $q_{1}=q_{2}=0$. Lemma 2.13 implies that $\operatorname{Ker} L_{I}^{\Psi}=\operatorname{Im} L_{I}^{\Phi}$. Proposition 2.8 for $n=2$ follows from Lemma 2.11.

We continue the proof for $n \geq 3$.

Lemma $2.17 q_{i}\left(e_{j}, e_{k}\right)=q_{j}\left(e_{k}, e_{i}\right)=q_{k}\left(e_{i}, e_{j}\right)$ for mutually distinct $i, j, k=$ $1, \ldots, n$.

Proof Since $i, j, k$ are mutually distinct, Lemma 2.15 implies

$$
\begin{aligned}
\frac{1}{3} \cdot\left[q_{i}, Q_{e_{j}}\right]\left(e_{k}, e_{k}, e_{k}\right) & =q_{i}\left(e_{k}, Q_{e_{j}}\left(e_{k}, e_{k}\right)\right)-Q_{e_{j}}\left(e_{k}, q_{i}\left(e_{k}, e_{k}\right)\right) \\
& =q_{i}\left(e_{k}, e_{j}\right)-\left\{\left\langle e_{k}, q_{i}\left(e_{k}, e_{k}\right)\right\rangle \cdot e_{j}-\left\langle e_{j}, q_{i}\left(e_{k}, e_{k}\right)\right\rangle \cdot e_{k}\right\} \\
& =q_{i}\left(e_{k}, e_{j}\right) .
\end{aligned}
$$


Similarly, we have $(1 / 3) \cdot\left[q_{j}, Q_{e_{i}}\right]\left(e_{k}, e_{k}, e_{k}\right)=q_{j}\left(e_{k}, e_{i}\right)$. Hence,

$$
q_{i}\left(e_{k}, e_{j}\right)-q_{j}\left(e_{k}, e_{i}\right)=\frac{1}{3} \cdot\left(\left[q_{i}, Q_{e_{j}}\right]-\left[q_{j}, Q_{e_{i}}\right]\right)\left(e_{k}, e_{k}, e_{k}\right)=0
$$

It implies $q_{i}\left(e_{j}, e_{k}\right)=q_{i}\left(e_{k}, e_{j}\right)=q_{j}\left(e_{k}, e_{i}\right)$. By permutations of indices $(i, j, k)$, we obtain that $q_{j}\left(e_{k}, e_{i}\right)=q_{k}\left(e_{i}, e_{j}\right)$.

Lemma 2.18 For $i, j, k=1, \ldots, n$,

$$
\begin{gathered}
q_{i}\left(e_{j}, e_{j}\right)=0, \\
\left\langle e_{i}, q_{i}\left(e_{j}, e_{k}\right)\right\rangle=\left\langle e_{j}, q_{i}\left(e_{j}, e_{k}\right)\right\rangle=\left\langle e_{k}, q_{i}\left(e_{j}, e_{k}\right)\right\rangle=0 .
\end{gathered}
$$

Proof For mutually distinct $i, j, k=1, \ldots, n$, we have

$$
\begin{aligned}
{\left[q_{i}, Q_{e_{j}}\right]\left(e_{j}, e_{k}, e_{k}\right)=} & \left\{q_{i}\left(e_{j}, Q_{e_{j}}\left(e_{k}, e_{k}\right)\right)+2 q_{i}\left(e_{k}, Q_{e_{j}}\left(e_{j}, e_{k}\right)\right)\right\} \\
& -\left\{Q_{e_{j}}\left(e_{j}, q_{i}\left(e_{k}, e_{k}\right)\right)+2 Q_{e_{j}}\left(e_{k}, q_{i}\left(e_{j}, e_{k}\right)\right)\right\} \\
= & \left\{q_{i}\left(e_{j}, e_{j}\right)+2 q_{i}\left(e_{k},-e_{k}\right)\right\} \\
& -\left\{-q_{i}\left(e_{k}, e_{k}\right)+2\left(\left\langle e_{k}, q_{i}\left(e_{j}, e_{k}\right)\right\rangle \cdot e_{j}-\left\langle e_{j}, q_{i}\left(e_{j}, e_{k}\right)\right\rangle \cdot e_{k}\right)\right\} \\
= & q_{i}\left(e_{j}, e_{j}\right)-q_{i}\left(e_{k}, e_{k}\right) \\
& \quad-2\left\langle e_{k}, q_{i}\left(e_{j}, e_{k}\right)\right\rangle \cdot e_{j}+2\left\langle e_{j}, q_{i}\left(e_{j}, e_{k}\right)\right\rangle \cdot e_{k},
\end{aligned}
$$

$\left[q_{j}, Q_{e_{i}}\right]\left(e_{j}, e_{k}, e_{k}\right)=\left\{q_{j}\left(e_{j}, Q_{e_{i}}\left(e_{k}, e_{k}\right)\right)+2 q_{j}\left(e_{k}, Q_{e_{i}}\left(e_{j}, e_{k}\right)\right)\right\}$

$$
\begin{aligned}
& \quad-\left\{Q_{e_{i}}\left(e_{j}, q_{j}\left(e_{k}, e_{k}\right)\right)+2 Q_{e_{i}}\left(e_{k}, q_{j}\left(e_{j}, e_{k}\right)\right)\right\} \\
& =\left\{q_{j}\left(e_{j}, e_{i}\right)+2 q_{j}\left(e_{k}, 0\right)\right\} \\
& \quad-\left\{\left\langle e_{j}, q_{j}\left(e_{k}, e_{k}\right)\right\rangle \cdot e_{i}-\left\langle e_{i}, q_{j}\left(e_{k}, e_{k}\right)\right\rangle \cdot e_{j}+2 Q_{e_{i}}\left(e_{k}, 0\right)\right\} \\
& =-\left\langle e_{j}, q_{j}\left(e_{k}, e_{k}\right)\right\rangle \cdot e_{i} .
\end{aligned}
$$

Since $\left[q_{i}, Q_{e_{j}}\right]-\left[q_{j}, Q_{e_{i}}\right]=0$, we obtain that $q_{i}\left(e_{j}, e_{j}\right)-q_{i}\left(e_{k}, e_{k}\right)$

$$
=-\left\langle q_{j}\left(e_{k}, e_{k}\right), e_{j}\right\rangle \cdot e_{i}+2\left\langle q_{i}\left(e_{j}, e_{k}\right), e_{k}\right\rangle \cdot e_{j}-2\left\langle q_{i}\left(e_{j}, e_{k}\right), e_{j}\right\rangle \cdot e_{k} .
$$

By taking the inner product of the with $e_{i}$ and $e_{j}$,

$$
\begin{aligned}
\left\langle e_{i}, q_{i}\left(e_{j}, e_{j}\right)\right\rangle-\left\langle e_{i}, q_{i}\left(e_{k}, e_{k}\right)\right\rangle & =-\left\langle e_{j}, q_{j}\left(e_{k}, e_{k}\right)\right\rangle, \\
\left\langle e_{j}, q_{i}\left(e_{j}, e_{j}\right)\right\rangle-\left\langle e_{j}, q_{i}\left(e_{k}, e_{k}\right)\right\rangle & =2\left\langle e_{k}, q_{i}\left(e_{j}, e_{k}\right)\right\rangle .
\end{aligned}
$$

The former equation for $i=1$ implies $\left\langle e_{j}, q_{j}\left(e_{k}, e_{k}\right)\right\rangle=0$ for any mutually distinct $j, k=2, \ldots, n$. By (17), the same equation holds for $j=1$ or $k=1$. Combined with (16), we obtain (18). 
Equations (16) and (20) imply $\left\langle e_{k}, q_{i}\left(e_{j}, e_{k}\right)\right\rangle=0$. By permutations of indices $(i, j, k)$ and Lemma 2.17, we obtain (19) for mutually distinct $i, j, k$. Equation (19) for other cases follows from Lemma 2.14 and (18).

Proposition 2.8 for $n=3$ follows from Lemma 2.18.

Proposition 2.19 If $n=3$, then $\operatorname{Ker} L_{I}^{\Psi}=\operatorname{Im} L_{I}^{\Phi}$.

Proof For $\left(q_{1}, q_{2}, q_{3}\right) \in \operatorname{Ker} L_{I}^{\Psi} \cap W$, (19) in Lemma 2.18 implies $q_{1}=q_{2}=q_{3}=0$ if $n=3$. By Lemma 2.13, we have $\operatorname{Ker} L_{I}^{\Psi}=\operatorname{Im} L_{I}^{\Phi}$. Proposition 2.8 for $n=3$ follows from Lemma 2.11.

The following lemma completes the proof for $n \geq 4$.

Lemma $2.20 q_{i}\left(e_{j}, e_{k}\right)=0$ for any $i, j, k=1, \ldots, n$.

Proof By Lemmas 2.14 and 2.18, it is enough to show $\left\langle e_{i}, q_{j}\left(e_{k}, e_{l}\right)\right\rangle=0$ for mutually distinct $i, j, k, l=1, \ldots, n$. Take mutually disjoint $i, j, k, l=1, \ldots, n$. Then, $\left[q_{i}, Q_{e_{j}}\right]\left(e_{k}, e_{l}, e_{l}\right)$

$$
\begin{aligned}
& =\left\{q_{i}\left(e_{k}, Q_{e_{j}}\left(e_{l}, e_{l}\right)\right)+2 q_{i}\left(e_{l}, Q_{e_{j}}\left(e_{k}, e_{l}\right)\right)\right\} \\
& \quad-\left\{Q_{e_{j}}\left(e_{k}, q_{i}\left(e_{l}, e_{l}\right)\right)+2 Q_{e_{j}}\left(e_{l}, q_{i}\left(e_{k}, e_{l}\right)\right)\right\} \\
& =\left\{q_{i}\left(e_{k}, e_{j}\right)+2 q_{i}\left(e_{l}, 0\right)\right\}-\left\{Q_{e_{j}}\left(e_{k}, 0\right)+2\left(\left\langle e_{l}, q_{i}\left(e_{k}, e_{l}\right)\right\rangle \cdot e_{j}-\left\langle e_{j}, q_{i}\left(e_{k}, e_{l}\right)\right\rangle \cdot e_{l}\right)\right\} \\
& =q_{i}\left(e_{j}, e_{k}\right)-2\left\langle e_{j}, q_{i}\left(e_{k}, e_{l}\right)\right\rangle \cdot e_{l} .
\end{aligned}
$$

Similarly, we obtain that

$$
\left[q_{j}, Q_{e_{i}}\right]\left(e_{k}, e_{l}, e_{l}\right)=q_{j}\left(e_{i}, e_{k}\right)-2\left\langle e_{i}, q_{j}\left(e_{k}, e_{l}\right)\right\rangle \cdot e_{l} .
$$

Since $\left[q_{i}, Q_{e_{j}}\right]-\left[q_{j}, Q_{e_{i}}\right]=0$,

$$
q_{i}\left(e_{j}, e_{k}\right)-q_{j}\left(e_{i}, e_{k}\right)=\left\{\left\langle e_{j}, q_{i}\left(e_{k}, e_{l}\right)\right\rangle-\left\langle e_{i}, q_{j}\left(e_{k}, e_{l}\right)\right\rangle\right\} \cdot e_{l} .
$$

By Lemma 2.17, $q_{i}\left(e_{j}, e_{k}\right)=q_{j}\left(e_{k}, e_{l}\right)$ and $q_{i}\left(e_{k}, e_{l}\right)=q_{k}\left(e_{l}, e_{i}\right)$. Hence, we have

$$
\left\langle e_{j}, q_{k}\left(e_{l}, e_{i}\right)\right\rangle=\left\langle e_{i}, q_{j}\left(e_{k}, e_{l}\right)\right\rangle .
$$

By take permutations of indices $(i, j, k, l)$,

$$
\left\langle e_{l}, q_{i}\left(e_{j}, e_{k}\right)\right\rangle=\left\langle e_{i}, q_{j}\left(e_{k}, e_{l}\right)\right\rangle=\left\langle e_{j}, q_{k}\left(e_{l}, e_{i}\right)\right\rangle=\left\langle q_{l}\left(e_{i}, e_{j}\right), e_{k}\right\rangle .
$$


On the other hand, we have

$\left[q_{i}, Q_{e_{j}}\right]\left(e_{j}, e_{k}, e_{l}\right)$

$$
\begin{aligned}
& =\left\{q_{i}\left(e_{j}, Q_{e_{j}}\left(e_{k}, e_{l}\right)\right)+q_{i}\left(e_{k}, Q_{e_{j}}\left(e_{l}, e_{j}\right)\right)+q_{i}\left(e_{l}, Q_{e_{j}}\left(e_{j}, e_{k}\right)\right)\right\} \\
& -\left\{Q_{e_{j}}\left(e_{j}, q_{i}\left(e_{k}, e_{l}\right)\right)+Q_{e_{j}}\left(e_{k}, q_{i}\left(e_{l}, e_{j}\right)\right)+Q_{e_{j}}\left(e_{l}, q_{i}\left(e_{j}, e_{k}\right)\right)\right\} \\
& =\left\{q_{i}\left(e_{j}, 0\right)+q_{i}\left(e_{k},-e_{l}\right)+q_{i}\left(e_{l},-e_{k}\right)\right\} \\
& -\left\{-q_{i}\left(e_{k}, e_{l}\right)+\left\langle e_{k}, q_{i}\left(e_{l}, e_{j}\right)\right\rangle \cdot e_{j}+\left\langle e_{l}, q_{i}\left(e_{j}, e_{k}\right)\right\rangle \cdot e_{j}\right\} \\
& =-q_{i}\left(e_{k}, e_{l}\right)-2\left\langle e_{j}, q_{i}\left(e_{k}, e_{l}\right)\right\rangle \cdot e_{j}, \\
& {\left[q_{j}, Q_{e_{i}}\right]\left(e_{j}, e_{k}, e_{l}\right)} \\
& =\left\{q_{j}\left(e_{j}, Q_{e_{i}}\left(e_{k}, e_{l}\right)\right)+q_{j}\left(e_{k}, Q_{e_{i}}\left(e_{l}, e_{j}\right)\right)+q_{j}\left(e_{l}, Q_{e_{i}}\left(e_{j}, e_{k}\right)\right)\right\} \\
& -\left\{Q_{e_{i}}\left(e_{j}, q_{j}\left(e_{k}, e_{l}\right)\right)+Q_{e_{i}}\left(e_{k}, q_{j}\left(e_{l}, e_{j}\right)\right)+Q_{e_{i}}\left(e_{l}, q_{j}\left(e_{j}, e_{k}\right)\right)\right\} \\
& =\left\{q_{j}\left(e_{j}, 0\right)+q_{j}\left(e_{k}, 0\right)+q_{j}\left(e_{l}, 0\right)\right\} \\
& -\left\{-\left\langle e_{i}, q_{j}\left(e_{k}, e_{l}\right)\right\rangle \cdot e_{j}+Q_{e_{i}}\left(e_{k}, 0\right)+Q_{e_{i}}\left(e_{l}, 0\right)\right\} \\
& =\left\langle e_{j}, q_{i}\left(e_{k}, e_{l}\right)\right\rangle \cdot e_{j} .
\end{aligned}
$$

Since $\left[q_{i}, Q_{e_{j}}\right]-\left[q_{j}, Q_{e_{i}}\right]=0$,

$$
q_{i}\left(e_{k}, e_{l}\right)+3 \cdot\left\langle e_{j}, q_{i}\left(e_{k}, e_{l}\right)\right\rangle \cdot e_{j}=0 .
$$

By taking the inner product with $e_{j}$, we have $\left\langle e_{j}, q_{i}\left(e_{k}, e_{l}\right)\right\rangle=0$. Hence,

$$
\left\langle e_{i}, q_{j}\left(e_{k}, e_{l}\right)\right\rangle=0
$$

by permuting indices $(i, j, k, l)$.

Now, we prove Proposition 2.8 for $n \geq 4$. The last lemma implies that $q_{1}=\cdots=q_{n}=0$ for any $\left(q_{1}, \ldots, q_{n}\right) \in \operatorname{Ker} L_{I}^{\Psi} \cap W$. By Lemma 2.13, we obtain that $\operatorname{Ker} L_{I}^{\Psi}=\operatorname{Im} L_{I}^{\Phi}$. Proposition 2.8 follows from Lemma 2.11.

\section{Classification of the standard actions}

In this section, we classify the standard $\Gamma_{n, k}$-actions up to smooth conjugacy. Let $O(n)$ be the orthogonal group of $\mathbb{R}^{n}$.

Proposition 3.1 For $B, B^{\prime} \in \mathrm{GL}_{n}(\mathbb{R}), \rho_{B}$ and $\rho_{B^{\prime}}$ are smoothly conjugate if and only if there exists $T \in O(n)$ and $c>0$ such that $B^{\prime}=(c T) B$.

Note that all standard $\Gamma_{n, k}$-actions are topologically conjugate to each other, ie there exists a homeomorphism $h$ of $S^{n}$ such that $\rho_{B^{\prime}}^{\gamma} \circ h=h \circ \rho_{B}^{\gamma}$ for any $\gamma \in \Gamma_{n, k}$. In 
fact, if $B^{\prime}=A B$ for some $A \in \mathrm{GL}_{n}(\mathbb{R})$, then the linear map $x \mapsto A x$ on $\mathbb{R}^{n}$ extends to a homeomorphism $h_{A}$ on $S^{n}$. It is easy to check that $\rho_{B^{\prime}}^{\gamma} \circ h_{A}=h_{A} \circ \rho_{B}^{\gamma}$ for any $\gamma=a, b_{1}, \ldots, b_{n}$. When $A=c T$ with $c>0$ and $T \in O(n)$, then $h_{A}$ is a diffeomorphism. Hence, $\rho_{B}$ and $\rho_{B^{\prime}}$ are smoothly conjugate in this case.

To prove the "only if" part of Proposition 3.1, we need a technical lemma. Recall that $Q_{v} \in \mathcal{S}^{2}\left(\mathbb{R}^{n}\right)$ is defined by

$$
Q_{v}(\xi, \eta)=\langle\xi, \eta\rangle \cdot v-\langle\xi, v\rangle \cdot \eta-\langle\eta, v\rangle \cdot \xi
$$

Lemma 3.2 Suppose that $A \circ Q_{v}=Q_{w} \circ(A, A)$ for $v, w \in \mathbb{R}^{n} \backslash\{0\}$ and $A \in \mathrm{GL}_{n}(\mathbb{R})$. Then, $A=c T$ for some $c>0$ and $T \in O(n)$.

Proof By a direct computation, we have

$$
\begin{aligned}
& Q_{w}(A \xi, A \xi)=\|A \xi\|^{2} \cdot w-2\langle A \xi, w\rangle \cdot A \xi \\
& A \circ Q_{v}(\xi, \xi)=\|\xi\|^{2} \cdot A v-2\langle\xi, v\rangle \cdot A \xi .
\end{aligned}
$$

Hence,

$$
\|A \xi\|^{2} \cdot w-\|\xi\|^{2} \cdot A v=2(\langle A \xi, w\rangle-\langle\xi, v\rangle) \cdot A \xi
$$

for any $\xi \in \mathbb{R}^{n}$. Put $\lambda=\left(2\langle A v, w\rangle-\|v\|^{2}\right) /\|A v\|^{2}$. Then, the equation for $\xi=v$ implies $w=\lambda A v$. By substituting it to (23), we have

$$
\left(\lambda\|A \xi\|^{2}-\|\xi\|^{2}\right) A v=2(\lambda\langle A \xi, A v\rangle-\langle\xi, v\rangle) \cdot A \xi
$$

Since $A$ is invertible, it implies that $\|A \xi\|=\lambda^{-1}\|\xi\|$ for any $\xi \in \mathbb{R}^{n} \backslash \mathbb{R} v$. Since $\mathbb{R}^{n} \backslash \mathbb{R} v$ is a dense subset of $\mathbb{R}^{n}$, the same holds for any $\xi \in \mathbb{R}^{n}$. Hence, there exists $T \in O(n)$ such that $A=\lambda^{-1} T$.

Proof of Proposition 3.1 It is enough to show the "only if" part. Suppose $\rho_{B}$ and $\rho_{B^{\prime}}$ are smoothly conjugate. Take a diffeomorphism $h$ of $S^{n}$ such that $\rho_{B^{\prime}}^{\gamma} \circ h=h \circ \rho_{B}^{\gamma}$ for any $\gamma \in \Gamma_{n, k}$. Since $\infty$ is the unique global fixed point of $\rho_{B}$ and $\rho_{B^{\prime}}$, the diffeomorphism $h$ fixes $\infty$. Recall $P_{B}$ and $P_{B^{\prime}}$ are the local $\Gamma_{n, k}$-actions defined by $P_{B}^{\gamma}=\bar{\phi} \circ \rho_{B}^{\gamma} \circ \bar{\phi}^{-1}$ and $P_{B^{\prime}}^{\gamma}=\bar{\phi} \circ \rho_{B^{\prime}}^{\gamma} \circ \bar{\phi}^{-1}$, where $\bar{\phi}(x)=\left(1 /\|x\|^{2}\right) \cdot x$. Put $H=\bar{\phi} \circ h \circ \bar{\phi}^{-1}$ and $A=D_{0}^{(1)} H$. Then, $P_{B^{\prime}}^{\gamma} \circ H=H \circ P_{B}^{\gamma}$, and hence,

$$
\begin{aligned}
D_{0}^{(1)} P_{B^{\prime}}^{\gamma} \circ D_{0}^{(2)} H+D_{0}^{(2)} P_{B^{\prime}}^{\gamma} \circ & (A, A) \\
& =A \circ D_{0}^{(2)} P_{B}^{\gamma}+D_{0}^{(2)} H \circ\left(D_{0}^{(1)} P_{B}^{\gamma}, D_{0}^{(1)} P_{B}^{\gamma}\right) .
\end{aligned}
$$

Since $P_{B}^{a}(x)=P_{B^{\prime}}^{a}(x)=k^{-1} x$, Equation (24) for $\gamma=a$ implies $k^{-1} D_{0}^{(2)} H=$ $k^{-2} D_{0}^{(2)} H$. Therefore, $D_{0}^{(2)} H=0$. Put $B=\left(v_{1}, \ldots, v_{n}\right)$ and $B^{\prime}=\left(w_{1}, \ldots, w_{n}\right)$. 
Since $D_{0}^{(2)} P_{B}^{b_{i}}=2 Q_{v_{i}}$ and $D_{0}^{(2)} P_{B^{\prime}}^{b_{i}}=2 Q_{w_{i}}$, (24) for $\gamma=b_{i}$ implies

$$
Q_{w_{i}} \circ(A, A)=A \circ Q_{v_{i}}
$$

for any $i=1, \ldots, n$. By Lemma 3.2, there exists $c>0$ and $T \in O(n)$ such that $A=c T$. Since $T$ preserves the inner product,

$$
\begin{aligned}
(c T) \circ Q_{v_{i}}(\xi, \eta) & =Q_{w_{i}}((c T) \xi,(c T) \eta) \\
& =c^{2}\left\{\langle T \xi, T \xi\rangle \cdot w_{i}-\left\langle T \xi, w_{i}\right\rangle \cdot T \eta-\left\langle T \eta, w_{i}\right\rangle \cdot T \xi\right\} \\
& =(c T) \circ\left\{\langle\xi, \eta\rangle \cdot\left(c T^{-1}\right) w_{i}-\left\langle\xi,\left(c T^{-1}\right) w_{i}\right\rangle \cdot \eta-\left\langle\eta,\left(c T^{-1}\right) w_{i}\right\rangle \cdot \xi\right\} \\
& =(c T) \circ Q_{c T^{-1} w_{i}}(\xi, \eta)
\end{aligned}
$$

for any $\xi, \eta \in \mathbb{R}^{n}$. It implies that $v_{i}=c T^{-1} w_{i}$ for any $i=1, \ldots, n$. Therefore, $B^{\prime}=\left(c^{-1} T\right) B$.

\section{References}

[1] E J Benveniste, Rigidity of isometric lattice actions on compact Riemannian manifolds, Geom. Funct. Anal. 10 (2000) 516-542 MR1779610

[2] L Burslem, A Wilkinson, Global rigidity of solvable group actions on $S^{1}$, Geom. Topol. 8 (2004) 877-924 MR2087072

[3] J Cantwell, L Conlon, An interesting class of $C^{1}$ foliations, Topology Appl. 126 (2002) 281-297 MR1934265

[4] D Damjanović, A Katok, Local rigidity of partially hyperbolic actions I. KAM method and $\mathbb{Z}^{k}$ actions on the torus, Ann. of Math. 172 (2010) 1805-1858 MR2726100

[5] D Fisher, First cohomology and local rigidity of group actions, to appear in Ann. of Math. (2) arXiv:0505520

[6] D Fisher, Local rigidity of group actions: past, present, future, from: "Dynamics, ergodic theory, and geometry", (B Hasselblatt, editor), Math. Sci. Res. Inst. Publ. 54, Cambridge Univ. Press (2007) 45-97 MR2369442

[7] D Fisher, G Margulis, Almost isometric actions, property (T), and local rigidity, Invent. Math. 162 (2005) 19-80 MR2198325

[8] D Fisher, G Margulis, Local rigidity of affine actions of higher rank groups and lattices, Ann. of Math. 170 (2009) 67-122 MR2521112

[9] N Guelman, I Liousse, Actions of Baumslag-Solitar groups on surfaces arXiv: 1004.2126

[10] N Guelman, I Liousse, $C^{1}$-actions of Baumslag-Solitar groups on $S^{1}$, Algebr. Geom. Topol. 11 (2011) 1701-1707 MR2821437 
[11] A Katok, B Hasselblatt, Introduction to the modern theory of dynamical systems, Encyclopedia of Mathematics and its Applications 54, Cambridge Univ. Press (1995) MR1326374

[12] A Katok, J Lewis, Local rigidity for certain groups of toral automorphisms, Israel J. Math. 75 (1991) 203-241 MR1164591

[13] A Katok, R J Spatzier, Differential rigidity of Anosov actions of higher rank abelian groups and algebraic lattice actions, Tr. Mat. Inst. Steklova 216 (1997) 292-319 MR1632177

[14] A E McCarthy, Rigidity of trivial actions of abelian-by-cyclic groups, Proc. Amer. Math. Soc. 138 (2010) 1395-1403 MR2578531

[15] A Navas, Groupes résolubles de difféomorphismes de l'intervalle, du cercle et de la droite, Bull. Braz. Math. Soc. 35 (2004) 13-50 MR2057043

[16] V Niţică, A Török, Local rigidity of certain partially hyperbolic actions of product type, Ergodic Theory Dynam. Systems 21 (2001) 1213-1237 MR1849607

[17] C Rivas, On spaces of Conradian group orderings, J. Group Theory 13 (2010) 337-353 MR2653523

[18] A Weil, Remarks on the cohomology of groups, Ann. of Math. 80 (1964) 149-157 MR0169956

[19] R J Zimmer, Lattices in semisimple groups and distal geometric structures, Invent. Math. 80 (1985) 123-137 MR784532

Department of Mathematics, Kyoto University

Kita-shirakawa Oiwake-cho, Sakyo, Kyoto 606-8502, Japan

asaoka-001@math.kyoto-u.ac.jp

Proposed: Benson Farb

Seconded: Leonid Polterovich, Yasha Eliashberg

Received: 14 September 2011

Revised: 6 April 2012 